\title{
The Phosphodiesterase Inhibitor Ensifentrine Reduces Production of Proinflammatory Mediators in Well Differentiated Bronchial Epithelial Cells by Inhibiting PDE4
}

\author{
Mark J. Turner, Nurlan Dauletbaev, Larry C. Lands, and (DJohn W. Hanrahan \\ Departments of Physiology (M.J.T., J.W.H.) and Pediatrics (N.D.) and Cystic Fibrosis Translational Research Centre (M.J.T., \\ L.C.L., J.W.H), McGill University, Montréal, Québec, Canada; Pediatric Respiratory Medicine, Montreal Children's Hospital, \\ Montréal, Québec, Canada (N.D., L.C.L.); Research Institute - McGill University Health Centre, Montréal, Québec, Canada \\ (L.C.L., J.W.H.); Department of Internal, Respiratory Translational Laboratory, Respiratory and Critical Care Medicine, \\ Philipps-University of Marburg, Marburg, Germany (N.D.); and Faculty of Medicine and Healthcare, al-Farabi Kazakh National \\ University, Almaty, Kazakhstan (N.D.)
}

Received April 30, 2020; accepted September 24, 2020

\begin{abstract}
Cystic fibrosis (CF) is caused by mutations in the cystic fibrosis transmembrane conductance regulator (CFTR) anion channel that impair airway salt and fluid secretion. Excessive release of proinflammatory cytokines and chemokines by CF bronchial epithelium during airway infection leads to chronic inflammation and a slow decline in lung function; thus, there is much interest in finding safe and effective treatments that reduce inflammation in CF. We showed previously that the cyclic nucleotide phosphodiesterase (PDE) inhibitor ensifentrine (RPL554; Verona Pharma) stimulates the channel function of CFTR mutants with abnormal gating and also those with defective trafficking that are partially rescued using a clinically approved corrector drug. PDE inhibitors also have known anti-inflammatory effects; therefore, we examined whether ensifentrine alters the production of proinflammatory cytokines in CF bronchial epithelial cells. Ensifentrine reduced the production of monocyte chemoattractant protein-1 and granulocyte monocyte colony-stimulating factor (GM-CSF) during challenge with interleukin- $1 \beta$. Comparing the effect of ensifentrine with milrinone and roflumilast, selective PDE3 and PDE4 inhibitors, respectively, demonstrated that the
\end{abstract}

anti-inflammatory effect of ensifentrine was mainly due to inhibition of PDE4. Beneficial modulation of GM-CSF was further enhanced when ensifentrine was combined with low concentrations of the $\beta_{2}$-adrenergic agonist isoproterenol or the corticosteroid dexamethasone. The results indicate that ensifentrine may have beneficial anti-inflammatory effects in CF airways particularly when used in combination with $\beta_{2}$ adrenergic agonists or corticosteroids.

\section{SIGNIFICANCE STATEMENT}

Airway inflammation that is disproportionate to the burden of chronic airway infection causes much of the pathology in the cystic fibrosis (CF) lung. We show here that ensifentrine beneficially modulates the release of proinflammatory factors in well differentiated CF bronchial epithelial cells that is further enhanced when combined with $\beta_{2}$-adrenergic agonists or lowconcentration corticosteroids. The results encourage further clinical testing of ensifentrine, alone and in combination with $\beta_{2}$-adrenergic agonists or low-concentration corticosteroids, as a novel anti-inflammatory therapy for CF.
This work was partially supported by a grant from Verona Pharma plc (UK) to J.W.H. and L.C.L. Verona Pharma did not participate in the design of experiments or interpretation of the results. Support was also provided by a grant from the Canadian Institutes of Health Research (PJT-156183) to J.W.H.

This work was previously presented at the 33rd Annual North American Cystic Fibrosis Conference; 2019 Oct; Nashville, TN. Citation: Turner MJ, Dauletbaev N, Lands LC, and Hanrahan, JW (2019) The phosphodiesterase inhibitor RPL554 reduces proinflammatory cytokine expression in welldifferentiated cystic fibrosis human airway epithelia. Pediatric Pulmonology 54(2): 159 .

https://doi.org/10.1124/jpet.120.000080.

\section{Introduction}

The cystic fibrosis transmembrane conductance regulator (CFTR) is a cAMP/protein kinase A-activated channel that plays an important role in the secretion of airway surface liquid, a microscopic layer of fluid that is essential for the innate defenses of the lungs (Elborn, 2016; Saint-Criq and Gray, 2017). Loss-of-function mutations in CFTR cause the autosomal recessive disease cystic fibrosis (CF), which is characterized by reduced airway salt and fluid secretion, increased susceptibility to bacterial infection, and chronic inflammation (Cantin et al., 2015; Roesch et al., 2018). The complex innate immune response to infection begins with

ABBREVIATIONS: ALI, air-liquid interface; CF, cystic fibrosis; CFTR, cystic fibrosis transmembrane conductance regulator; $\mathrm{Cl}$, confidence interval; COPD, chronic obstructive pulmonary disease; CRE, CAMP response element; GM-CSF, granulocyte macrophage colony-stimulating factor; GR, glucocorticoid receptor; IL, interleukin; $I_{s c}$, short-circuit current; M-BEGM, modified bronchial epithelial cell growth medium; MCP-1, monocyte chemoattractant protein-1; PDE, cyclic nucleotide phosphodiesterase; pHBE, primary human bronchial epithelial; qPCR, quantitative polymerase chain reaction; WT, wild type. 
epithelial secretion of proinflammatory cytokines and chemokines. These include interleukin (IL)-8 and tumor necrosis factor alpha (TNF- $\alpha$ ), which mediate the chemotaxis and neutrophil activation (Mukaida, 2003), granulocyte macrophage colony-stimulating factor (GM-CSF), which stimulates proliferation and maturation of granulocytes and macrophages (Becher et al., 2016), and monocyte chemoattractant protein-1 (MCP-1), which recruits monocytes (Deshmane et al., 2009). The resulting influx of immune cells to the site of infection helps combat infection; however, in CF it produces a neutrophil-dominated, unopposed inflammation that leads to irreversible lung damage.

A major goal of CF pharmacotherapy is to correct the basic defect in the airways, i.e., restore CFTR-dependent ion and fluid transport. However, drugs that reduce excessive inflammation and promote its resolution after infection could also provide benefit. Several anti-inflammatory drugs, including high-dose ibuprofen, azithromycin, and inhaled corticosteroids, have been clinically tested in patients with $\mathrm{CF}$ but were found to be of limited effectiveness or unsafe (Ross et al., 2009; Ratjen et al., 2012; Principi et al., 2015; Lands and Stanojevic, 2019). Clinical trials for several other anti-inflammatory drugs for $\mathrm{CF}$ are ongoing, and there is clearly a need for new and better treatments that modulate airway inflammation without compromising the ability to fight infection.

Cyclic nucleotide phosphodiesterases (PDEs) are a family of enzymes that degrade cAMP, cGMP, and other cyclic nucleotides. Eleven members of the cyclic nucleotide PDE family are expressed in humans (Maurice et al., 2014). Inhibition of PDE3 and PDE4 in human bronchial epithelial cells elevates intracellular cAMP levels and increases CFTR activity (AlNakkash and Hwang, 1999; Cobb et al., 2003; Barnes et al., 2005; Liu et al., 2005; Blanchard et al., 2014; Tyrrell et al., 2015). Recently, we demonstrated that the PDE inhibitor ensifentrine (RPL554; Verona Pharma, London, UK) stimulates both wildtype CFTR and the disease causing mutant R117H-CFTR when endogenously expressed in well differentiated primary human bronchial epithelial cells (Turner et al., 2016). We further observed that ensifentrine activates the CFTR mutants R334W-, T338I-, S549R-, and G551D-CFTR when expressed in the Fisher rat thyroid cell model (Turner et al., 2020). These data indicate that activation of $\mathrm{cAMP} /$ protein kinase A signaling by ensifentrine has therapeutic potential for CFTR mutants with residual trafficking of CFTR to the plasma membrane.

PDE3 inhibitors, including ensifentrine, induce bronchodilation in chronic obstructive pulmonary disease (COPD) and asthma (Myou et al., 1999; Singh et al., 2018), whereas PDE3 and PDE4 inhibitors have previously been used as antiinflammatory and immunomodulatory drugs for several respiratory diseases (Banner and Page, 1995; Abbott-Banner and Page, 2014). However, there is surprisingly little information on the anti-inflammatory effects of PDE inhibitors in CF. Therefore, we assessed whether ensifentrine could elicit anti-inflammatory effects in CF airways by measuring proinflammatory cytokine production in $\mathrm{CF}$ and non-CF airway epithelia. Our results indicate that ensifentrine causes small but significant decreases in GM-CSF and MCP-1 production and has prominent anti-inflammatory effects when combined with a low concentration of dexamethasone. These results suggest that, in addition to increasing CFTR channel activity, PDE inhibitors have beneficial anti-inflammatory effects in CF when used in combination with corticosteroids.

\section{Methods}

Cell Culture. The CFBE410 ${ }^{-}$cell lines overexpressing wild-type (WT) CFTR (CFBE410 ${ }^{-}$WT) or F508del CFTR (CFBE41o ${ }^{-}$F508del), kindly provided by Drs. Jeong Hong and Eric Sorscher (Emory University), were passaged in Eagle's Minimum Essential Media supplemented with FBS (10\%), L-glutamine (2 mM), penicillin (100 $\mathrm{U} / \mathrm{ml})$, and streptomycin $(100 \mu \mathrm{g} / \mathrm{ml})$. For experiments, the cells were seeded on fibronectin-coated, $0.33 \mathrm{~cm}^{2}$ permeable Transwell supports (Corning) (80,000 cells/well). After 24 hours, the apical medium was removed, and the basolateral medium was replaced with air-liquid interface (ALI) medium (Lonza) to induce differentiation. The medium was changed every 48 hours, and cells were grown at the air-liquid interface for 7 days prior to experiments. Primary human bronchial epithelial cells from three non-CF donors (one male, two female) and three F508del/F508del patients (two male, one female) were isolated by the Primary Airway Cell Biobank in the Cystic Fibrosis Translational Research Centre at McGill University using lung tissue provided by the biobank of respiratory tissues at the Centre Hospitalier de l'Université de Montréal after informed, written consent from donors. All procedures were approved by the Institutional Review Board of McGill University (A08-M70-14B). Cells were isolated using methods similar to those described by Fulcher et al. (2005). They were seeded on type IV collagencoated, $0.33 \mathrm{~cm}^{2}$ permeable Transwell supports (Corning). The apical media were removed after 3 days, and cells were differentiated at the air-liquid interface for 4 weeks prior to experiments.

Cell Treatment and Sample Collection. Differentiated CFBE41oand well differentiated primary human bronchial epithelial (pHBE) cells were washed $3 \times$ with PBS and incubated for 6 hours in modified bronchial epithelial cell growth medium (M-BEGM; Lonza) lacking retinoic acid, hydrocortisone, and antibiotics. These factors were omitted as previous studies had shown that they influence cytokine

\begin{tabular}{|c|c|c|c|c|}
\hline Day 0 & Day 1 & Day 8 & Day 9 & Day 10 \\
\hline $\begin{array}{l}\text { CFBE41o- cells } \\
\text { seeded }\end{array}$ & $\begin{array}{l}\text { Cells put at the air-liquid } \\
\text { interface and cultured in } \\
\text { ALI media }\end{array}$ & $\begin{array}{l}\text { Cells incubated in } \mathrm{M} \text { - } \\
\text { BEGM for } 6 \mathrm{~h} \text { before } \\
\text { addition of vehicle or drug }\end{array}$ & $\begin{array}{l}\text { Addition of pro-inflammatory } \\
\text { challenge. After } 8 \mathrm{~h} \text {, RNA is } \\
\text { collected from some filters }\end{array}$ & $\begin{array}{l}\text { Collection of } \\
\text { supernatant }\end{array}$ \\
\hline
\end{tabular}

Day 0

Day 4

pHBE cells

seeded
Day 32

Cells put at the air-liquid interface and cultured in ALI media

Day 33

Day 34

Cells incubated in $\mathrm{M}$ BEGM for $6 \mathrm{~h}$ before addition of vehicle or drug

Fig. 1. Protocols showing cell differentiation, treatment, proinflammatory challenge, and sample collection. 
production and expression (Mathew and Sharma, 2000; Zhao and Ding, 2018). Transwell cultures were treated basolaterally with pharmacological agents for 18 hours, then challenged basolaterally with the proinflammatory agonists. Cells were exposed to IL- $1 \beta$ (10 ng/ml) or flagellin from Pseudomonas aeruginosa $(5 \mathrm{ng} / \mathrm{ml})$ for 8 hours for RNA extraction, or 24 hours when collecting supernatant. These concentrations were used based on reported concentrations of IL-1 $\beta$ and flagellin found in sputum collected from patients with CF (Osika et al., 1999; Eickmeier et al., 2010; Balloy et al., 2014; Eckrich et al., 2017) and are consistent with our previous studies (Dauletbaev et al., 2010). A basolateral challenge was used to 1) mimic IL-1 $\beta$ release from bloodborne immune cells, notably macrophages, which are elevated in the bronchial mucosae of CF children (Tang et al., 2012) and 2) enable flagellin to activate its cognate receptor toll-like receptor 5 . Toll-like receptor 5 is mainly expressed on the basolateral membrane of bronchial epithelia (Shikhagaie et al., 2014) and induces a larger effect on cytokine gene expression when added basolaterally compared with when added apically (Hybiske et al., 2004). A schematic of the experimental design is shown in Fig. 1.

Measurements of cAMP, Cytokines, and Chemokines. Intracellular cAMP ([cAMP]i) was measured using the Direct cAMP ELISA kit (Enzo Life Sciences) according to the manufacturer's instructions. MCP-1, GM-CSF, IL-10, IL-23, and IL-33 were measured in the basolateral medium bathing air-liquid interface cultures of CFBE410 ${ }^{-}$cells and well differentiated pHBE cells using the Milliplex Map kit (EMD Millipore) and the manufacturer's instructions. Briefly, samples were added to fluorescent-coded magnetic bead sets coated with specific capture antibodies (MagPlex-C Microspheres) that allowed multiple cytokines to be quantified in one sample. After adding the detection antibody and streptavidin-phycoerythrin reporter molecule, samples were read using the Luminex analyzer MAGPIX and analyzed using the Milliplex Analyst 5.1 Software. Some GM-CSF measurements were also made using the OptEIA Human GM-CSF ELISA set (BD Biosciences) as per the manufacturer's instructions. IL-8 was measured in the basolateral medium of well differentiated CFBE410 ${ }^{-}$cells and pHBE cells using the OptEIA Human IL-8 ELISA set (BD Biosciences) following methods from the manufacturer.

RNA Purification and Reverse Transcription. Cells were lysed with RNA Lysis Buffer from the Zymogen Quick RNA kit and RNA was purified by following the manufacturer's instructions. One hundred nanogram of RNA were reverse transcribed by adding $4 \mu \mathrm{l}$ of $5 \times$ All-In-One MasterMix (ABM) in a reaction volume of $20 \mu \mathrm{l}$. The reaction was carried out using the following protocol: $25^{\circ} \mathrm{C}$ for 10 minutes, $42^{\circ} \mathrm{C}$ for 15 minutes, and $85^{\circ} \mathrm{C}$ for 5 minutes.

Quantitative Polymerase Chain Reaction. Quantitative polymerase chain reactions (qPCRs) were performed by combining $1.5 \mu \mathrm{l}$ cDNA with $10 \mu \mathrm{M}$ forward and reverse primer targeting the gene of interest (Intergrated DNA Technologies) and $10 \mu \mathrm{l}$ of SsoAdvanced Universal SYBR Green Supermix (Bio-Rad) made up to a $20 \mu \mathrm{l}$ reaction volume using molecular grade $\mathrm{H}_{2} \mathrm{O}$. Polymerase chain reactions were performed using a BioRad CFX96 Real-Time PCR Detection System with the following protocol: $95^{\circ} \mathrm{C}$ for 2 minutes followed by 40 cycles of $95^{\circ} \mathrm{C}$ for 10 seconds, $60^{\circ} \mathrm{C}$ for 30 seconds before performing a melt curve analysis. To quantify gene expression, $\Delta \Delta \mathrm{CT}$ analysis was performed by normalizing the expression of genes of interest to that of the reference gene Hypoxanthine-guanine phosphoribosyltransferase (HPRT1). The following primer sequences were used: MCP-1, forward, 5'-GCCACCTTCATTCC-3', reverse, 5'-AGC AGCCTCTGCACTGAGATCTTC-3'; GM-CSF, forward, 5'-TGACAA GCAGAAAGTCCTTCAG-3' ${ }^{\prime}$, reverse, $5^{\prime}$-CAGCCTCACCAAGCTCAA G-3'; IL-8, forward, 5' -CGCCAACACAGAAATTATTGTAAAG-3', reverse, $5^{\prime}$-AACTTCTCCACAACCCTCTG-3'; HPRT1, forward, 5' -GCG ATGTCAATAGGACTCCAG-3', reverse, 5'-TTGTTGTAGGATATG CCCTTGA-3'.

Short-Circuit Current Measurements. For measurements of short circuit current, cells were cultured at the air-liquid interface in ALI medium for 7 days before being washed $3 \times$ with PBS and incubated for 48 hours in M-BEGM. Short-circuit current $\left(\mathrm{I}_{\mathrm{sc}}\right)$ measurements were then performed as previously described (Turner et al., 2020).

Solutions and Reagents. All reagents were purchased from Sigma Aldrich unless otherwise stated. Ensifentrine was obtained from Verona Pharma. CFTR inh $^{-172}$ was obtained courtesy of Dr. Robert Bridges (Rosalind Franklin University) and Cystic Fibrosis Foundation Therapeutics Inc. For $\mathrm{I}_{\mathrm{sc}}$ measurements, the basolateral solution contained (millimolar) $115 \mathrm{NaCl}, 25 \mathrm{NaHCO}_{3}, 1.2 \mathrm{MgCl}_{2}, 1.2 \mathrm{CaCl}_{2}$, $1.2 \mathrm{~K}_{2} \mathrm{HPO}_{4}, 2.4 \mathrm{KH}_{2} \mathrm{PO}_{4}$, and 10 D-glucose; $\mathrm{pH}$ 7.4. The apical solution contained (millimolar) 1.2 NaCl, NaGluconate, $25 \mathrm{NaHCO}_{3}, 1.2 \mathrm{MgCl}_{2}$, $4 \mathrm{CaCl}_{2}, 1.2 \mathrm{~K}_{2} \mathrm{HPO}_{4}, 2.4 \mathrm{KH}_{2} \mathrm{PO}_{4}$, and 10 D-glucose; $\mathrm{pH} 7.4$.

Statistical Analysis. Data were analyzed using GraphPad Prism 6.0 (GraphPad Software). $N$ indicates the number of independent experiments performed, whereas $n$ signifies the number of technical replicates in one experiment. The sample sizes and number of experiments were set prior to the data being obtained. All data sets were used for statistical analyses. The Students $t$ test was used to assess the statistical significance of differences between data of interest. A $P$ value of $<0.05$ was considered significant.

\section{Results}

Ensifentrine Elevates Intracellular cAMP Levels and Stimulates Wild-Type CFTR in Well Differentiated CFBE41o $^{-}$Cells. We have previously demonstrated that ensifentrine concentration-dependently stimulates wild-type CFTR overexpressed in CFBE410 ${ }^{-}$cells or endogenously
A

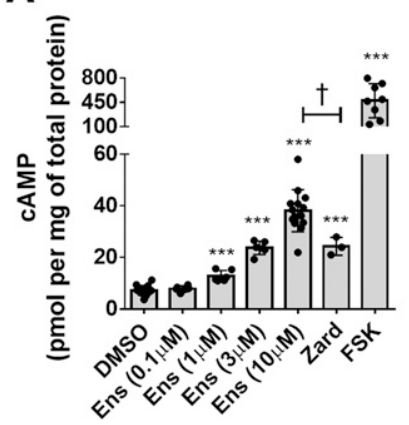

B

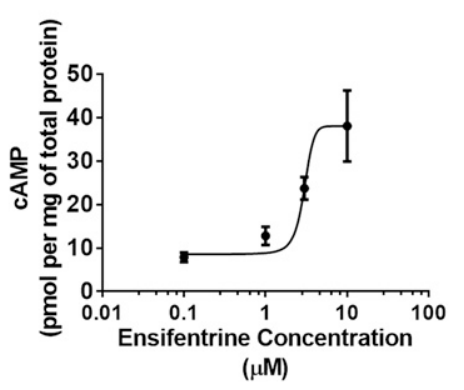

C

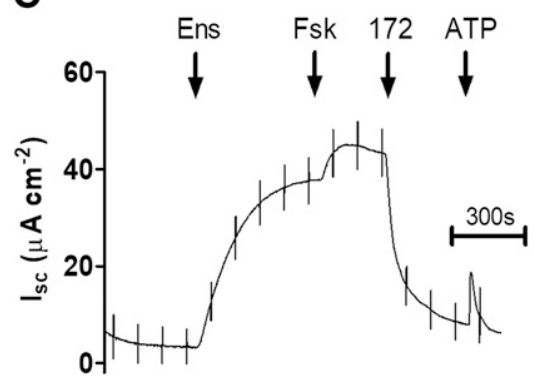

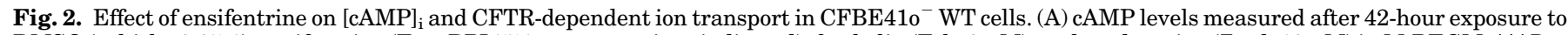

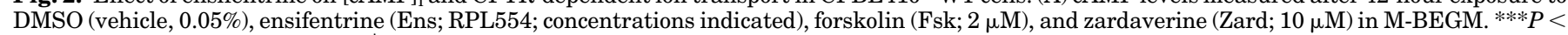

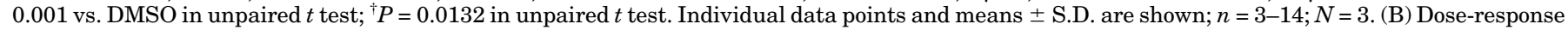

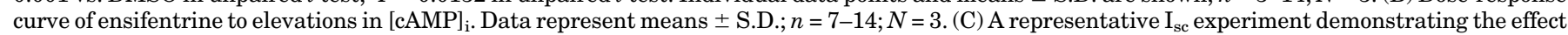

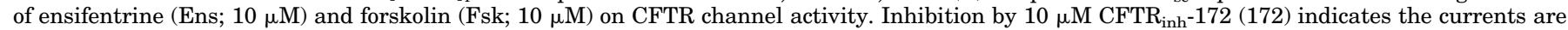
CFTR-dependent. $n=6 ; N=2$. 
TABLE 1

List of cytokines analyzed in well differentiated human airway epithelia supernatant using MultiPlex ELISAs

\begin{tabular}{|c|c|c|}
\hline Cytokine & Function & References \\
\hline MCP-1 & $\begin{array}{l}\text { Expressed in airway epithelia, MCP-1 regulates infiltration of immune cells } \\
\text { (i.e., monocytes and macrophages) to site of injury. Found to be significantly } \\
\text { higher in sputum and serum of patients with CF vs. healthy persons }\end{array}$ & $\begin{array}{l}\text { Rao et al., 2000; Lundien et al., 2002; } \\
\text { Brennan et al., 2009; Velikova et al., } 2018\end{array}$ \\
\hline GM-CSF & $\begin{array}{l}\text { Produced by airway epithelia, GM-CSF stimulates the production of immune cells, } \\
\text { including neutrophils and monocytes. Found to be elevated in patients with CF } \\
\text { specifically with chronic } P \text {. aeruginosa lung infection }\end{array}$ & Cox et al., 1991; Jensen et al., 2006 \\
\hline IL-8 & $\begin{array}{l}\text { Proinflammatory cytokine produced by airway epithelial cells and is elevated in } \\
\text { bronchiolar lavage fluid and sputum of patients with CF vs. healthy persons }\end{array}$ & $\begin{array}{l}\text { Dean et al., 1993; Bonfield et al., 1995b; } \\
\text { Sagel et al., } 2001\end{array}$ \\
\hline IL-10 & $\begin{array}{l}\text { Anti-inflammatory cytokine that acts to reduce gene expression of proinflammatory } \\
\text { cytokines. Shown to be downregulated in CF airway epithelia }\end{array}$ & Bonfield et al., 1995a \\
\hline IL-23 & Proinflammatory cytokine expressed in airway epithelia & Lee et al., 2017 \\
\hline IL-33 & $\begin{array}{l}\text { Cytokine expressed in airway epithelia that induces the production of } \\
\text { proinflammatory cytokines from mast cells, eosinophils, and helper } \mathrm{T} \text { cells }\end{array}$ & $\begin{array}{l}\text { Moussion et al., 2008; Préfontaine et al., 2010; } \\
\text { Drake and Kita, 2017; Lee et al., } 2017\end{array}$ \\
\hline
\end{tabular}

expressed in pHBE cells isolated from non-CF donors, suggesting that ensifentrine-induced elevation of $[\mathrm{cAMP}]_{i}$ activates CFTR (Turner et al., 2016). For the present study, to be compatible with cytokine assays, $\mathrm{CFBE} 410^{-}$cells had to be cultured in different media than in previous studies (i.e., ALI medium for differentiation, followed by M-BEGM for experiments). To confirm that ensifentrine elevates intracellular cAMP and stimulates CFTR in CFBE410 ${ }^{-}$cells under these conditions, cAMP was measured in cells that were grown for 7 days in ALI medium and then treated for 6 hours with M-BEGM prior to addition of cAMP-elevating agonists for a further 42 hours. Figure 2 shows that ensifentrine concentration-dependently increased $[\mathrm{cAMP}]_{\mathrm{i}}$ and, at $10 \mu \mathrm{M}$, induced a $5.3 \pm 0.9$ fold increase in $[\mathrm{cAMP}]_{\mathrm{i}}$ compared with vehicle control [95\% confidence interval [CI]: 4.4-6.1; $P=$ $0.0002 ; n=14$; Fig. $2 \mathrm{~A}$ ] confirming that under these conditions inhibiting PDE3/4 with ensifentrine was sufficient to elevate $[\mathrm{cAMP}]_{i}$. Although the dual PDE3/4 inhibitor zardarverine significantly increased $[\mathrm{cAMP}]_{\mathrm{i}} 3.4 \pm 0.7$ fold compared with control conditions (95\% CI: $2.3-4.4 ; P=0.0132 ; n=3$ ), this was significantly lower than the effect of ensifentrine at the same concentration, indicating that ensifentrine is more potent than zardarverine (Fig. 2A). When cells were cultured for 7 days in ALI medium followed by 2 days in M-BEGM, then mounted in Ussing chambers, acute addition of ensifentrine increased the mean $\mathrm{I}_{\mathrm{sc}}$ by $35.6 \pm 4.5 \mu \mathrm{A} \mathrm{cm}{ }^{-2}$ (95\% CI:

A

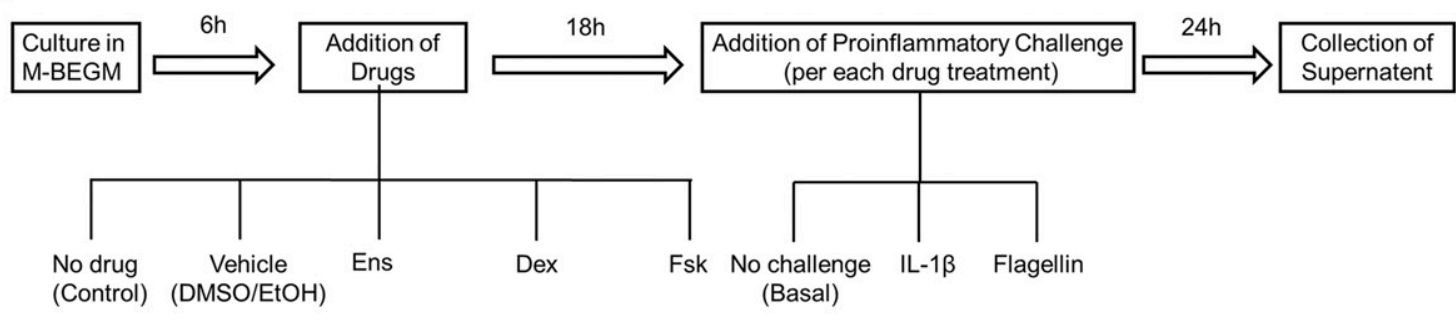

B

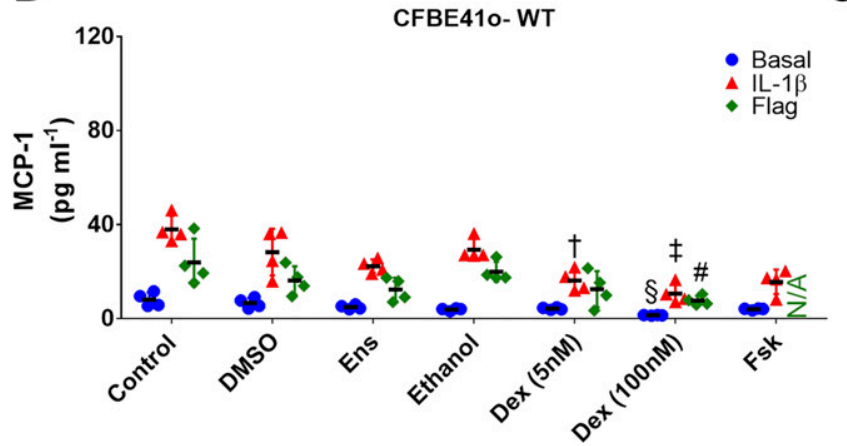

C

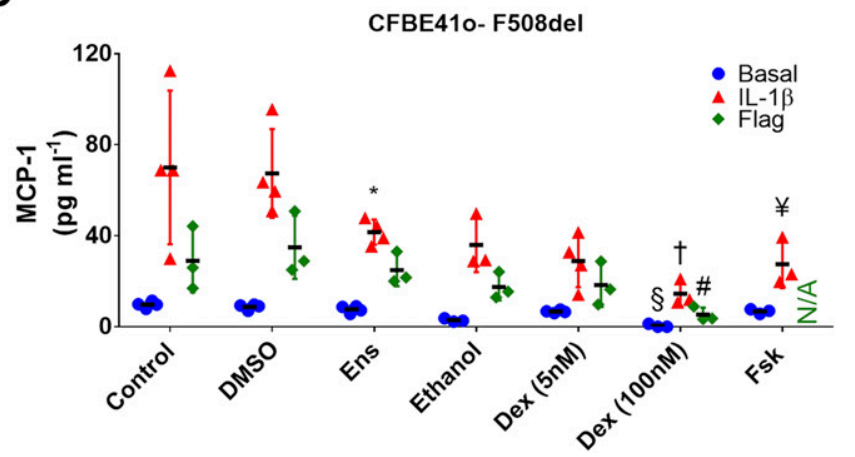

Fig. 3. Effects of ensifentrine on basal and stimulated MCP-1 production in CFBE41o ${ }^{-}$WT and F508del cells. (A) Schematic of protocol for treating CFBE410 ${ }^{-}$cells cultured at the air-liquid interface for 7 days. Incubation in M-BEGM was followed by treatment with vehicle (DMSO for ensifentrine and forskolin; 0.05\%, ethanol for dexamethasone; 0.05\%), ensifentrine (Ens; $10 \mu \mathrm{M}$ ), dexamethasone (Dex; 5 or $100 \mathrm{nM}$ ) or forskolin (Fsk; $2 \mu \mathrm{M}$ ) for 18 hours. Cells were then challenged with the proinflammatory stimuli IL-1 $\beta$ (10 ng/ml) or flagellin (Flag; $5 \mathrm{ng} / \mathrm{ml})$ for 24 hours. This was followed by supernatant collection and MCP-1 level quantification by MultiPlex ELISA. (B) Summary of MCP-1 levels in CFBE410- WT cells. ${ }^{\S} P=0.0001$ in unpaired $t$ test vs. vehicle in basal conditions; ${ }^{\dagger} P=0.0067 ;{ }^{\ddagger} P=0.0009$ in unpaired $t$ tests vs. vehicle after IL- $1 \beta$ challenge; ${ }^{\#} P=0.0020$ in unpaired $t$ test vs. vehicle after flagellin challenge. (C) Summary of MCP-1 levels in CFBE41o ${ }^{-}$F508del cells. ${ }^{\S} P=0.0178$ in unpaired $t$ test vs. vehicle in basal conditions; ${ }^{*} P=0.0324$ in unpaired $t$ test; ${ }^{\dagger} P=0.0479$ in unpaired $t$ test and ${ }^{¥} P=0.0247$ in unpaired $t$ test vs. vehicle after IL-1 $\beta$ challenge. \#P=0.0339 in unpaired $t$ test vs. vehicle after flagellin challenge. Individual data points and means \pm S.D. are shown; $N=3$ to 4 . 
30.8-40.4 $\mu \mathrm{A} \mathrm{cm}^{-2} ; n=6$; Fig. $2 \mathrm{~B}$ ), a response that was only slightly increased by maximal forskolin stimulation. Together, these observations demonstrate that ensifentrine causes robust elevation of cAMP levels and triggers cAMP-dependent downstream signaling, including activation of CFTR, in CFBE41o cells under these culture conditions.

Production of MCP-1, GM-CSF, and IL-8 in Well Differentiated CFBE41o- Cells Is Upregulated by Proinflammatory Challenge. We next wanted to establish that well differentiated cells in our experimental model upregulate production of inflammatory cytokines when exposed to proinflammatory challenges. For this, the cells were cultured in ALI medium for 7 days prior to treatment with M-BEGM for 6 hours, then exposed to vehicle for 18 hours before proinflammatory challenge with either IL- $1 \beta$ or flagellin for 8 or 24 hours. The outcomes were expression of respective mRNAs ( 8 hours of stimulation) or production of protein in the supernatant (24 hours of stimulation). The cytokines of interest were studied using SinglePlex or MultiPlex ELISAs (Table 1). Of the six cytokines examined, only MCP-1, GM-CSF, and IL-8 were upregulated by inflammatory challenge. In contrast, levels of IL-10, IL-23, or IL-33 were all either below the detection limit for the assay or were detectable but unaltered by the proinflammatory stimuli (data not shown). We therefore focused the subsequent experiments on measuring MCP-1, GM-CSF, and IL-8 responses to ensifentrine treatment.

Ensifentrine Significantly Reduces Production of MCP-1 in Stimulated CFBE41o- F508del Cells. We first assessed the effect of ensifentrine on MCP-1 production in $\mathrm{CFBE} 10^{-}$cells in both basal and proinflammatory conditions, in which cells were stimulated basolaterally for 24 hours with either IL-1 $\beta$ or $P$. aeruginosa flagellin (Fig. 3A). We also compared the effect of ensifentrine with the cAMPelevating agonist forskolin and two different concentrations of the anti-inflammatory drug dexamethasone (5 or $100 \mathrm{nM}$ ). Under basal conditions, both CFBE410 ${ }^{-}$WT and CFBE410 F508del cells produced similar levels of MCP-1 (Fig. 3, B and C). We observed IL- $1 \beta$ and flagellin to increase MCP-1 levels $4.7 \pm 2.7$ fold (95\% CI: $2.5-6.9 ; P=0.00009 ; n=4)$ and $3.0 \pm 1.7$ fold (95\% CI: $1.0-4.9 ; P=0.0242 ; n=4)$ in CFBE410 ${ }^{-}$WT cells, respectively, and 7.2 \pm 3.6 fold (95\% CI: $2.9-11.4 ; P=0.0118$; $n=4)$ and $3.0 \pm 1.5$ fold ( $95 \% \mathrm{CI}: 0.5-5.4 ; P=0.0356 ; n=3)$ in CFBE410 ${ }^{-}$F508del cells, respectively (Fig. 3, B and C). Neither vehicle (DMSO or ethanol) altered MCP-1 levels under basal or proinflammatory conditions in the cell lines examined. Although ensifentrine did not affect MCP-1 release under basal conditions, it did reduce MCP-1 levels by $42 \% \pm$ $19 \%$ in $\mathrm{CFBE} 410^{-}$F508del cells when challenged with IL-1 $\beta$ (95\% CI: 20\%-64\%; $P=0.0324 ; n=4$; Fig. 3C). Interestingly, forskolin caused a similar decrease in MCP-1, suggesting that the anti-inflammatory effect of ensifentrine during IL-1 $\beta$ challenge was mediated by elevation of $[\mathrm{cAMP}]_{\mathrm{i}}$. The antiinflammatory drug dexamethasone had a concentrationdependent effect such that the higher concentration of $100 \mathrm{nM}$ was significantly anti-inflammatory in both cell lines under basal and proinflammatory conditions (Fig. 3, B and C). Further studies examined modulation of MCP-1 mRNA levels in ensifentrine-treated CFBE410 ${ }^{-}$cells. In this experiment, the cells were pretreated for 18 hours with a vehicle (DMSO) or ensifentrine, challenged with IL- $1 \beta$ or flagellin for 8 hours, and then collected for mRNA analyses (Fig. 4A). Under control

A
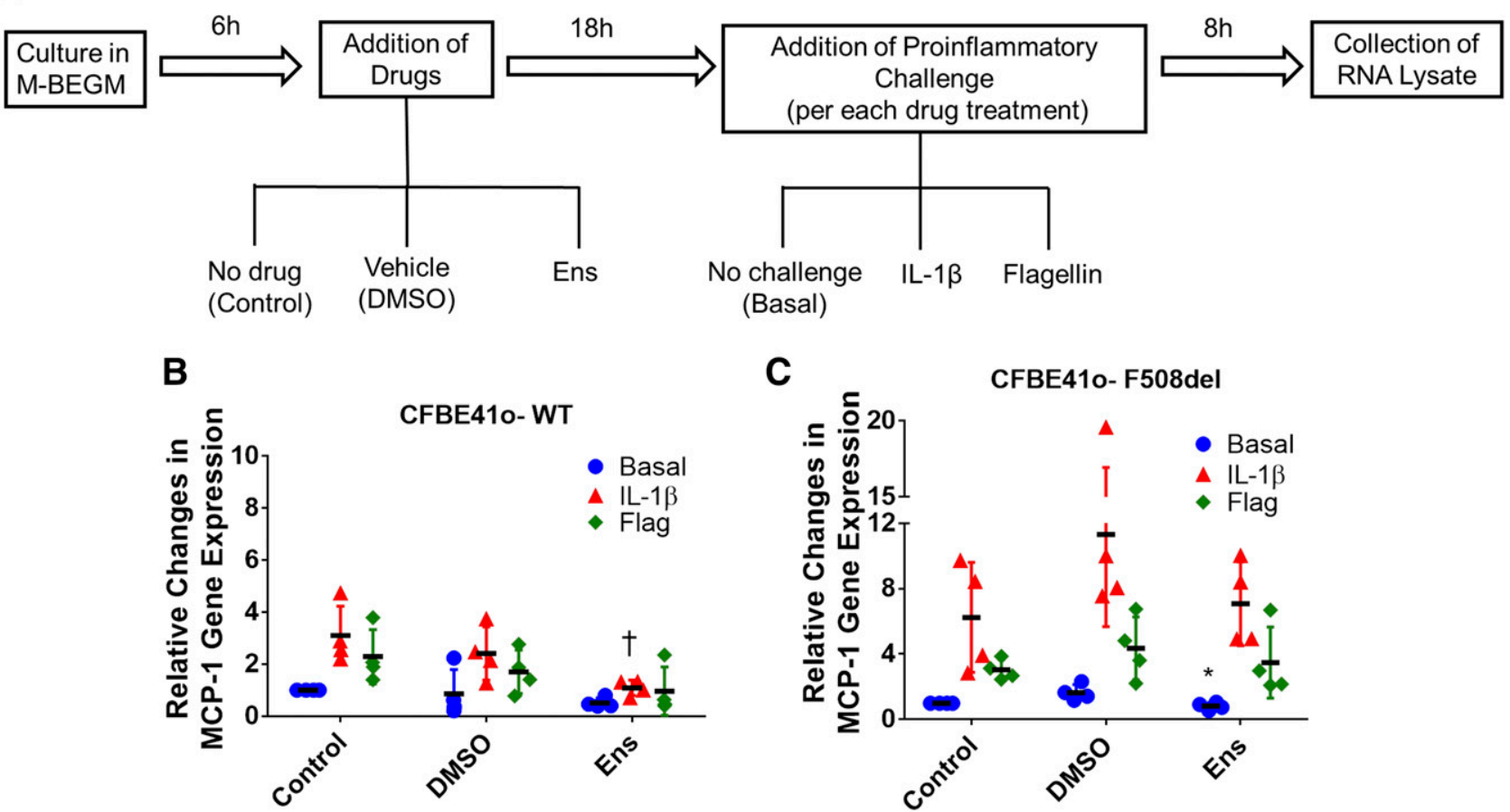

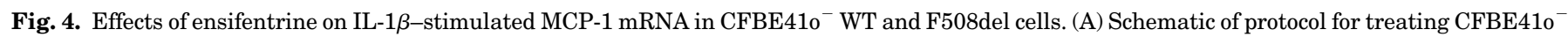

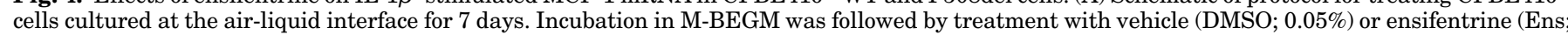

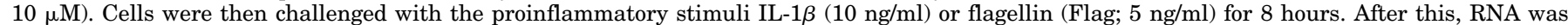

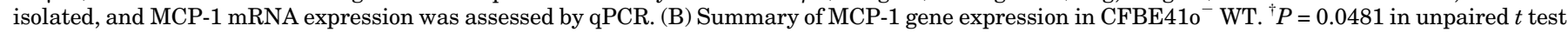

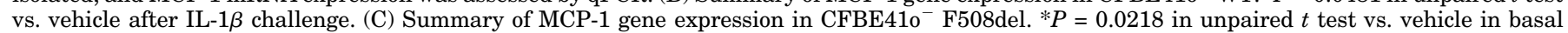
conditions. Individual data points and means \pm S.D. are shown; $N=3$ to 4 . 
A

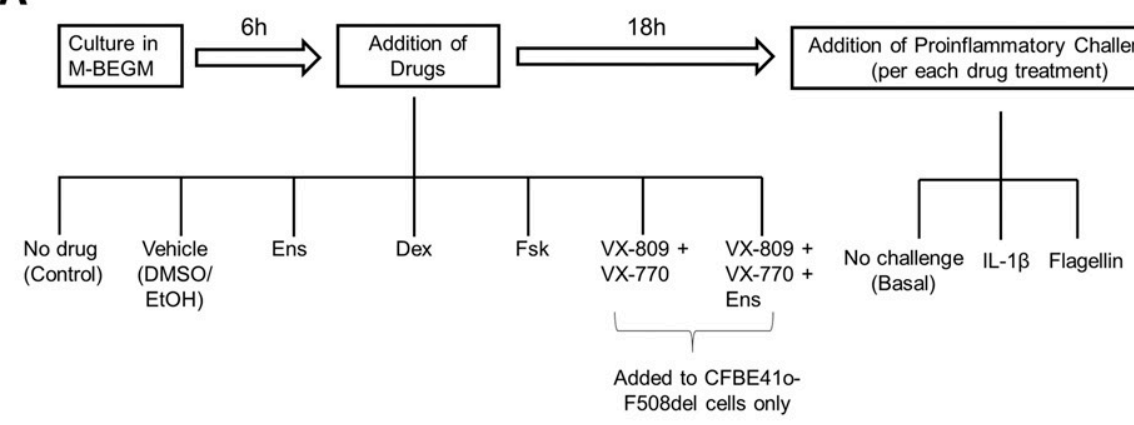

B

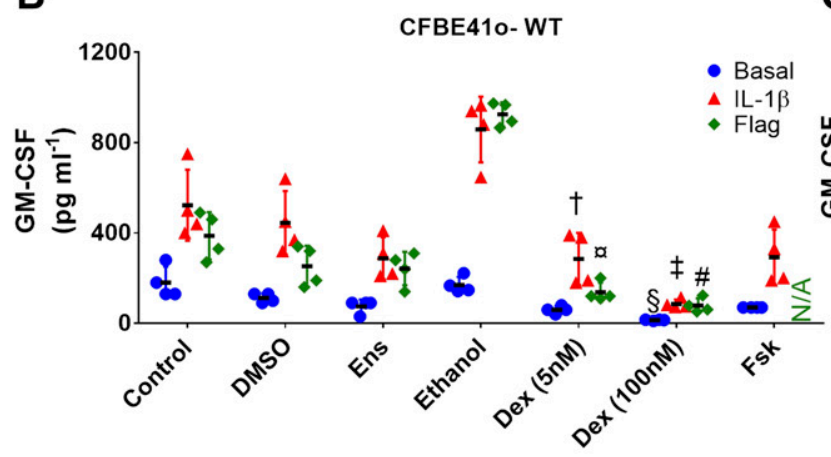

C CFBE410- F508del

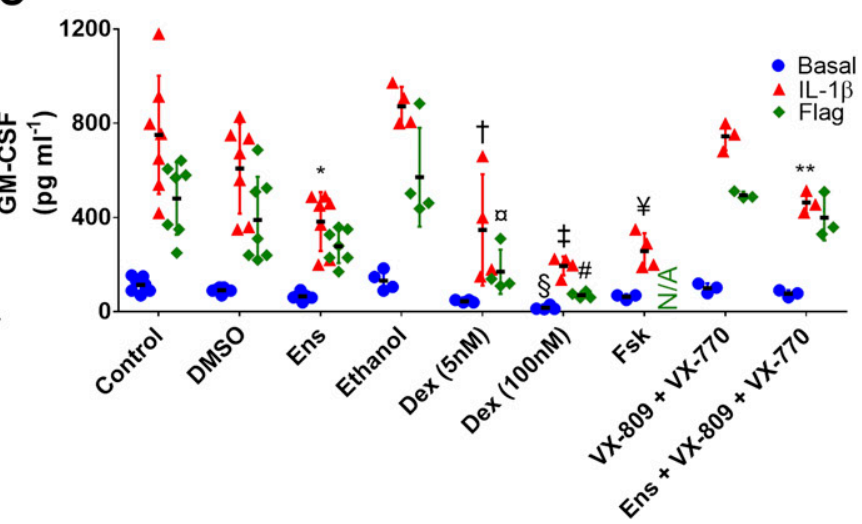

Fig. 5. Effects of ensifentrine on basal and stimulated GM-CSF production in CFBE410 ${ }^{-}$WT and F508del cells. (A) Schematic of protocol for treating CFBE $410^{-}$cells cultured at the air-liquid interface for 7 days. Incubation in M-BEGM was followed by treatment with vehicle (DMSO for ensifentrine and forskolin; $0.05 \%$, ethanol for dexamethasone; 0.05\%), ensifentrine (Ens; $10 \mu \mathrm{M}$ ), dexamethasone (Dex; 5 or $100 \mathrm{nM}$ ), or forskolin (Fsk; $2 \mu \mathrm{M})$ for 18 hours. Some CFBE41o ${ }^{-}$F508del cells were also treated with VX-809 $(1 \mu \mathrm{M})$ and VX-770 $(100 \mathrm{nM})$ in the absence or presence of ensifentrine. Cells were then challenged with the proinflammatory stimuli IL-1 $\beta(10 \mathrm{ng} / \mathrm{ml})$ or flagellin (Flag; $5 \mathrm{ng} / \mathrm{ml})$ for 24 hours. This was followed by supernatant collection and GM-CSF level quantification by MultiPlex ELISA. (B) Summary of GM-CSF levels in CFBE41o ${ }^{-}$WT cells. ${ }^{\S} P=0.0001$ in unpaired $t$ test vs. vehicle in basal conditions; ${ }^{\dagger} P=0.0008 ;{ }^{\ddagger} P=0.00004$ in unpaired $t$ tests vs. vehicle in IL- $1 \beta$ challenged conditions; ${ }^{\circ} P=0.0000004 ;{ }^{\#} P=0.0000002$ in unpaired $t$ tests vs. vehicle in flagellin challenged conditions. (C) Summary of GM-CSF levels in CFBE410 ${ }^{-}$F508del cells. ${ }^{\S} P=0.0018$ in unpaired $t$ test vs. vehicle in basal conditions; $* P=0.0227 ;{ }^{\sharp} P=0.0073 ;{ }^{\dagger} P=0.0058 ;{ }^{\ddagger} P=0.000006$ in unpaired $t$ test vs. vehicle in IL- $1 \beta$ challenged conditions; ${ }^{\circ} P=$ $0.0130 ;{ }^{\#} P=0.0031$ in unpaired $t$ tests vs. vehicle in flagellin challenged conditions. ${ }^{* *} P=0.0031$ in unpaired $t$ test vs. VX-809 + VX-770 alone in IL-1 $\beta$ challenged conditions. Individual data points and means \pm S.D. are shown; $N=3$ to 4 .

conditions, IL-1 $\beta$ and flagellin increased MCP-1 mRNA expression in both cell lines similar to the increases in protein (Fig. 4, B and C vs. Fig. 3, B and C). In CFBE410 ${ }^{-}$WT cells challenged with IL-1 $\beta$, there was a small reduction in MCP-1 mRNA level after ensifentrine treatment compared with cells treated with vehicle $(P=0.0481 ; n=4 ;$ Fig. $4 \mathrm{~B})$, indicative of transcriptional regulation by ensifentrine. In CFBE $410^{-}$ F508del cells, ensifentrine only modulated basal MCP-1 mRNA levels (Fig. 4C) and did not affect the upregulation by respective stimuli (Fig. 4C).

Ensifentrine Reduces GM-CSF Production and mRNA Levels in CFBE41o- ${ }^{-}$Cells. The MultiPlex ELISA panel was used to further test ensifentrine modulation of another inflammatory cytokine, namely GM-CSF. A schematic of this protocol shown in Fig. 5A was identical to that for MCP-1. Under basal conditions, both cell lines tested had similar levels of GM-CSF (Fig. 5, B and C). IL- $1 \beta$ induced a $2.9 \pm 1.4$ fold increase in GM-CSF production in CFBE41o ${ }^{-}$ WT cells (95\% CI: $1.2-4.6 ; P=0.073 ; n=4$ ) and a significantly higher $7.0 \pm 4.1$ fold increase in GM-CSF production in CFBE410 ${ }^{-}$F508del cells (95\% CI: $2.1-11.8 ; P=0.0121$ vs. control; $P=0.0031$ vs. CFBE410 ${ }^{-}$WT; $n=4$; Fig. 5 , B and C). Meanwhile, flagellin caused a $2.1 \pm 1.0$ fold increase in CFBE $410^{-}$WT cells $(95 \%$ CI: $0.9-3.3 ; P=0.0167 ; n=4)$ and a $3.9 \pm 1.9$ fold in CFBE410 ${ }^{-}$F508del cells (95\% CI: $1.6-6.1$; $P=0.0069$; Fig. 5, B and C). We were surprised to observe that ethanol enhanced the GM-CSF production in response to proinflammatory stimuli, in CFBE410 ${ }^{-}$WT cells (Fig. 5B) suggesting that ethanol can modulate inflammatory responses in airway epithelia. Importantly, ensifentrine caused a $37.1 \% \pm$ $21.5 \%$ decrease in IL- $1 \beta$-stimulated GM-CSF production in CFBE41o- F508del cells compared with vehicle control, indicating anti-inflammatory effects (95\% CI: $16.7 \%-57.4 \% ; P=$ 0.0227; $n=7$; Fig. 5C), whereas forskolin elicited a similar effect, consistent with the anti-inflammatory action of ensifentrine being mediated by elevation of $[\mathrm{cAMP}]_{i}$ (Fig. 5C). Dexamethasone reduced GM-CSF levels under basal and proinflammatory conditions in a concentration-dependent manner in both WT and F508del cells, serving as an antiinflammatory positive control. We also tested whether there was any effect on GM-CSF production in CFBE41o- F508del cells by treatment with the CFTR-modulating drugs VX-809 and VX-770, which mimic the clinical drug Orkambi (Wainwright et al., 2015). VX-809 + VX-770 did not significantly affect basal or stimulated GM-CSF production in CFBE410 F508del cells, nor did they enhance the inhibition by ensifentrine (Fig. 5C). Therefore, partial correction of F508del-CFTR does not alter GM-CSF production in CF epithelia. 

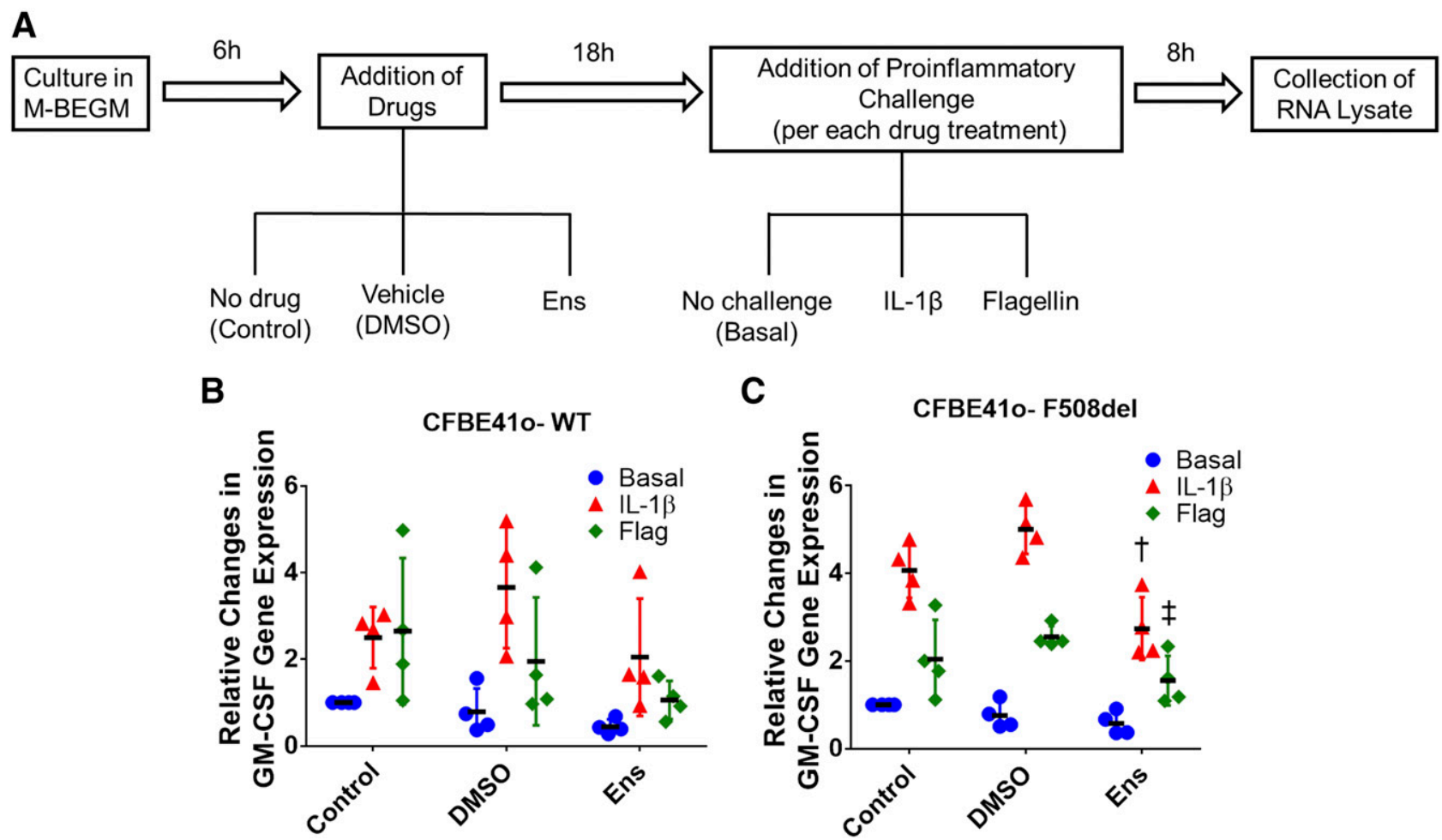

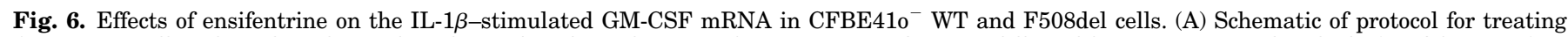

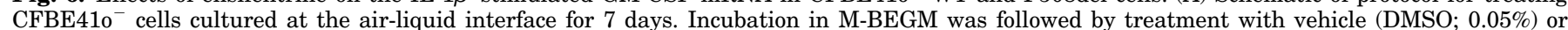

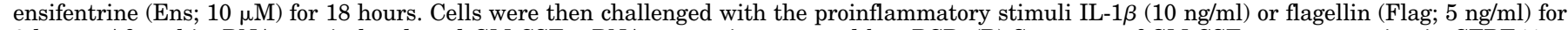

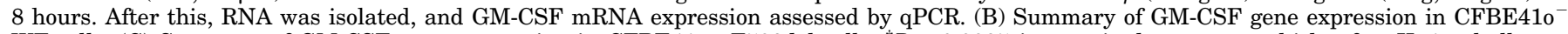

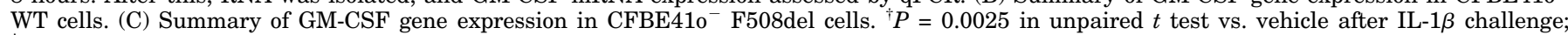
${ }^{\ddagger} P=0.0177$ in unpaired $t$ test, vs. vehicle after flagellin challenge. Individual data points and means $\pm \mathrm{S} . \mathrm{D}$. are shown; $N=3$ to 4 .

We next assessed the expression of GM-CSF mRNA in CFBE410 ${ }^{-}$cells with or without ensifentrine, and in the presence or absence of proinflammatory stimuli (Fig. 6A). Increases in GM-CSF mRNA induced by IL- $1 \beta$ and flagellin closely mirrored increases in protein production (Fig. 6, B and $\mathrm{C}$ vs. Fig. 5, B and C). Furthermore, in both tested cell lines, ensifentrine reduced the IL- $1 \beta$-stimulated elevation of GMCSF mRNA levels, with this inhibition reaching statistical significance in CFBE410 ${ }^{-}$F508del cells (Fig. 6C). Therefore, ensifentrine mediates its effects on GM-CSF production at the transcriptional level.

Ensifentrine has No Effect on IL-8 Protein and mRNA Expression in CFBE41o ${ }^{-}$Cells. As the last inflammatory cytokine in our MultiPlex ELISA panel, we quantified the production of IL-8 in CFBE410- cell lines under basal and stimulated conditions. Cells were pretreated with vehicle, ensifentrine, different concentrations of dexamethasone or forskolin before a 24-hour proinflammatory challenge and IL-8 quantification (Fig. 7A). Interestingly, basal IL-8 production was higher under control conditions in CFBE410 ${ }^{-}$ WT cells than in CFBE410 ${ }^{-}$F508del cells. However, IL-1 $\beta$ induced similar elevations in IL-8 production in CFBE410- WT and CFBE410 ${ }^{-}$F508del cells with respective fold increases of $20.6 \pm 6.9$ (95\% CI: $12.5-28.8 ; P=0.0008 ; n=4)$ and $23.4 \pm 8.9$ (95\% CI: $12.9-34.0 ; P=0.0000004 ; n=4$; Fig. 7, B and C). Likewise, flagellin also induced similar respective fold increases in IL-8 production of $15.7 \pm 6.9$ (95\% CI: 8.8-22.7; $P=0.00002 ; n=4)$ and $12.8 \pm 4.2(95 \% \mathrm{CI}: 7.9-17.7 ; P=$ $0.0021 ; n=4$; Fig. $7, \mathrm{~B}$ and $\mathrm{C}$ ). Note that the robust effect of proinflammatory stimuli on IL-8 production contrasts with the relatively small upregulation of MCP-1 and GM-CSF (Fig. 7, B and C vs. Figs. 3, B and C and 5, B and C). As was the case with GM-CSF, CFBE410- WT cells under proinflammatory challenge had enhanced IL-8 production in the presence of ethanol consistent with ethanol modulating cytokine production in these cells. In both cell lines, neither IL-1 $\beta$ nor flagellin-stimulated upregulation of IL-8 was altered by ensifentrine treatment. Dexamethasone induced only a small downregulation of IL-8 at $5 \mathrm{nM}$ with a greater effect at $100 \mathrm{nM}$ (Fig. 7, B and C). These concentrationdependent effects resemble those observed for MCP-1 and GM-CSF. Similar to GM-CSF, VX-809 + VX-770 did not significantly affect basal or stimulated IL-8 production in CFBE410 ${ }^{-}$F508del cells, nor did they modulate the response to ensifentrine (Fig. 7C). Therefore, partial correction of F508del does not modulate IL-8 production in CF epithelia.

We also examined ensifentrine modulation of IL-8 mRNA transcript levels during stimulation (Fig. 8A). As expected, challenge with both IL- $1 \beta$ or flagellin increased IL- 8 mRNA expression but this upregulation was not significantly altered by treatment with ensifentrine (Fig. 8, B and C).

Effects of Ensifentrine on MCP-1, GM-CSF, and IL-8 Production in pHBE Cells. We next examined responses in primary bronchial epithelial cells isolated from $\mathrm{CF}$ and nonCF donors. The overall experimental design is presented in Fig. 9A. When comparing cytokine production in control conditions in non-CF pHBE cells and CF pHBE cells, we observed IL-1 $\beta$-stimulated MCP-1 and GM-CSF levels to be 
A

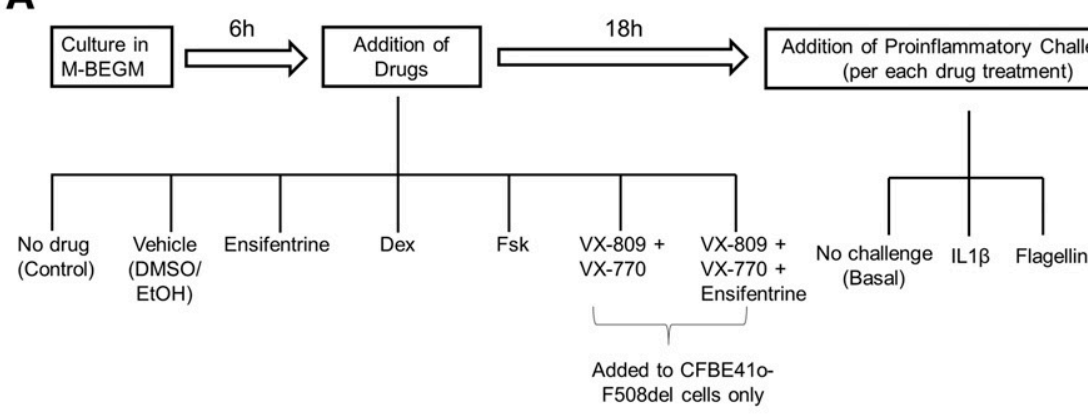

B

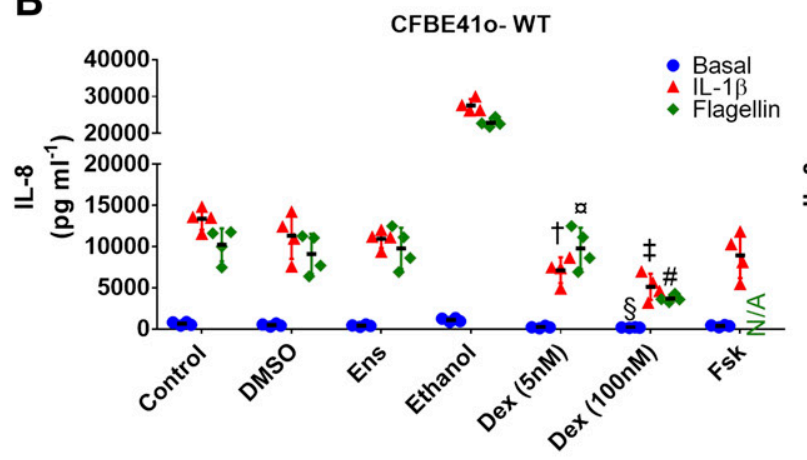

C
CFBE410- F508del

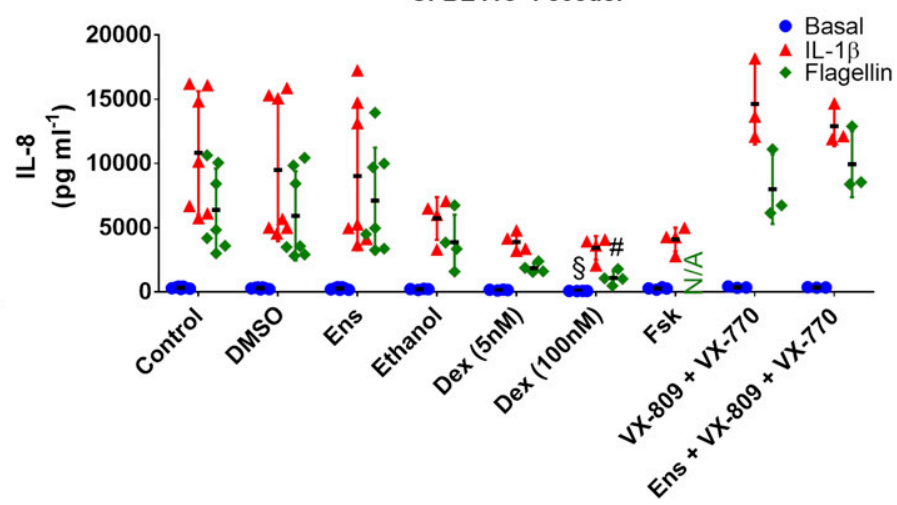

Fig. 7. Effects of ensifentrine on basal and stimulated production of IL-8 in CFBE410 ${ }^{-}$WT and F508del cells. (A) Schematic of protocol for treating CFBE $410^{-}$cells cultured at the air-liquid interface for 7 days. Incubation in M-BEGM was followed by treatment with vehicle (DMSO for ensifentrine and forskolin; $0.05 \%$, ethanol for dexamethasone; 0.05\%), ensifentrine (Ens; $10 \mu \mathrm{M}$ ), dexamethasone (Dex; 5 or $100 \mathrm{nM}$ ), or forskolin (Fsk; $2 \mu \mathrm{M}$ ) for 18 hours. Some CFBE41o ${ }^{-}$F508del cells were also treated with VX-809 $(1 \mu \mathrm{M})$ and VX-770 $(100 \mathrm{nM})$ in the absence or presence of ensifentrine. Cells were then challenged with the proinflammatory stimuli IL- $1 \beta(10 \mathrm{ng} / \mathrm{ml})$ or flagellin $(5 \mathrm{ng} / \mathrm{ml})$ for 24 hours. This was followed by supernatant collection and IL--8 level quantification by MultiPlex ELISA. (B) Summary of IL-8 levels in CFBE410 ${ }^{-}$WT cells. ${ }^{\S} P=0.0002$ in unpaired $t$ test vs. vehicle in basal conditions; ${ }^{\dagger} P=0.0195 ; \doteqdot P=0.0021$ in unpaired $t$ tests vs. vehicle in IL- $1 \beta$ challenged conditions; ${ }^{\#} P=0.0004$ in unpaired $t$ test vs. vehicle in flagellin challenged conditions. (C) Summary of IL-8 levels in CFBE41o- F508del cells. ${ }^{\S} P=0.005$ in unpaired $t$ test vs. vehicle in basal conditions; ${ }^{\sharp} P=0.0441$ in unpaired $t$ test vs. vehicle in flagellin challenged conditions. Individual data points and means \pm S.D. are shown; $N=3$ to 4 .

2-fold higher in the CF pHBE cells, suggesting that $\mathrm{CF}$ airway epithelia mount a greater inflammatory response when challenged with proinflammatory stimuli (compare Fig. 9, B and C and Fig. 9, D and E). Production of MCP-1, GM-CSF, and IL-8 was largely unaffected by ensifentrine treatment, whether it was used alone or in combination with VX-809 + VX-770, although there was a tendency for ensifentrine to reduce IL- $1 \beta$-stimulated GM-CSF production in CF pHBE cells both when used alone and with VX-809 + VX-770 (Fig. 9E) indicating a small positive effect of ensifentrine. The fact that VX-809 + VX-770 alone did not cause the same trend suggests that merely correcting F508del CFTR is not sufficient to dampen proinflammatory cytokine production. A low concentration of dexamethasone $(5 \mathrm{nM})$ also did not have significant anti-inflammatory effects but tended to reduce IL$1 \beta$-stimulated GM-CSF (Fig. 9, D-G). The lack of a robust effect of $5 \mathrm{nM}$ dexamethasone in pHBE cells is consistent with results in CFBE410 ${ }^{-}$cells.

Ensifentrine Mediates Its Effects through Inhibition of PDE4. Although the effect was small, ensifentrine was able to significantly reduce IL- $1 \beta$-stimulated MCP-1 and GMCSF production in well differentiated CFBE410 ${ }^{-}$F508del cells. Ensifentrine is marketed as a dual PDE3/4 inhibitor but is 3440 times more potent toward PDE3 than PDE4 (BoswellSmith et al., 2006). We compared IL- $1 \beta$-stimulated GM-CSF levels in $\mathrm{CFBE} 410^{-}$cells treated with ensifentrine, the PDE4 selective inhibitor roflumilast, the PDE3 selective inhibitor milrinone and the dual PDE3/4 inhibitor zardaverine to gain further insights into the mechanism of action (Fig. 10A). In both CFBE410 ${ }^{-}$WT and CFBE410 ${ }^{-}$F508del cells, we observed significant reductions in IL- $1 \beta$-stimulated GM-CSF production by ensifentrine and roflumilast but there was no effect of milrinone (Fig. 10, B and C). Zardaverine elicited significant effects in CFBE $410^{-}$WT cells but, surprisingly, not in CFBE410 ${ }^{-}$F508del cells. Together, these data demonstrate that PDE4 inhibition underlies the anti-inflammatory effects on ensifentrine in CFBE410 ${ }^{-}$cells.

Combining Ensifentrine with Isoproterenol or Low-Concentration Dexamethasone Causes Enhanced Downregulation of GM-CSF in CFBE410 ${ }^{-}$Cells. We next explored whether the anti-inflammatory effects of ensifentrine could be enhanced by combination therapies. Several studies have demonstrated that PDE inhibition can enhance the ability of corticosteroids and/or $\beta_{2}$-adrenergic agonists to modulate the expression of genes under the regulation of the glucocorticoid response element and cAMP response element (CRE) (Moodley et al., 2013; BinMahfouz et al., 2015; Reddy et al., 2020). We therefore tested the effects of combining ensifentrine with low concentrations of dexamethasone and/or isoproterenol on IL- $1 \beta$-stimulated GM-CSF production in CFBE410 ${ }^{-}$F508del cells. A schematic of the experimental protocol is shown on Fig. 11A. As previously shown, addition of 
A

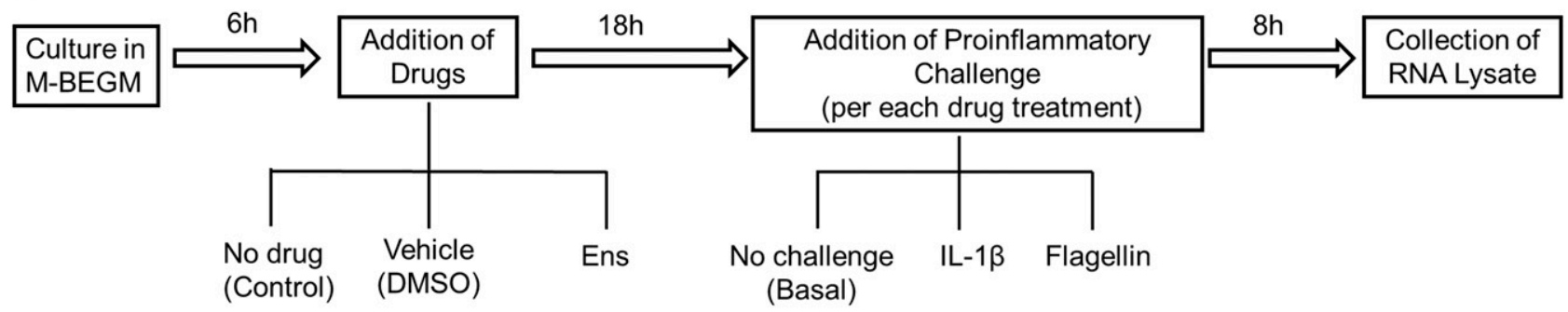

B

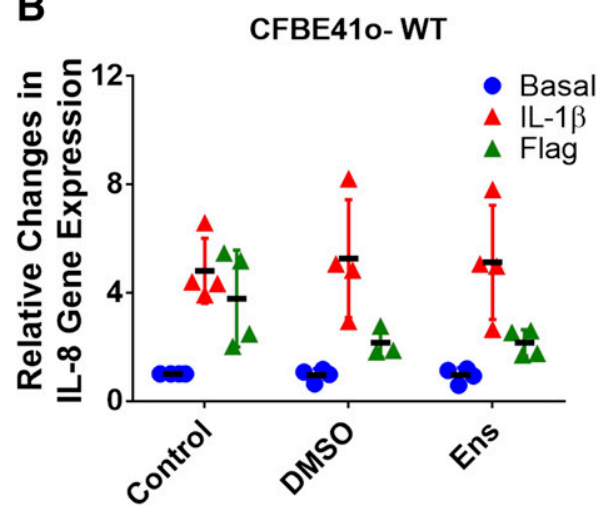

C

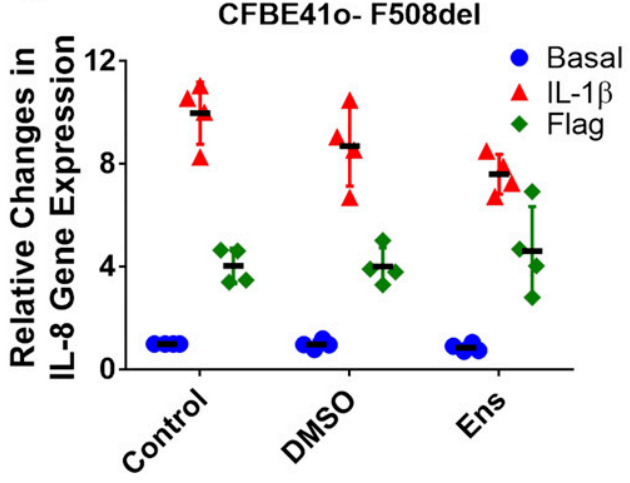

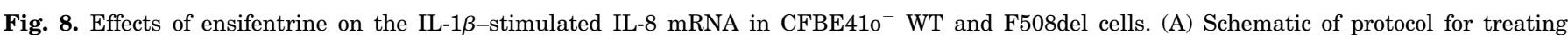

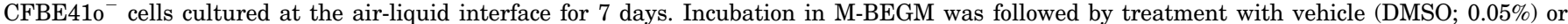

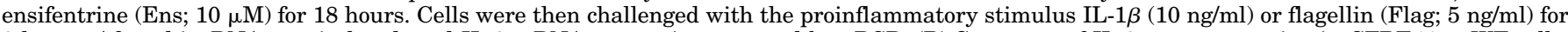

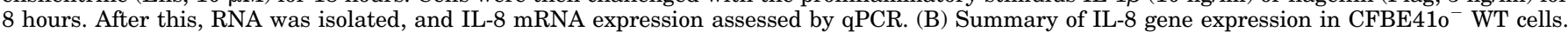
(C) Summary of IL-8 gene expression in CFBE410 ${ }^{-}$F508del. Individual data points and means \pm S.D. are shown; $N=3$ to 4.

ensifentrine induced a small but significant $22.5 \% \pm 16.5 \%$ reduction on IL-1 $\beta$-stimulated GM-CSF levels compared with vehicle control (95\% CI: $6.8 \%-38.1 \% ; P=0.0170 ; n=7$; Fig. 11B). However, in the presence of isoproterenol, this was further enhanced to a $47.6 \% \pm 5.1 \%$ reduction $(95 \% \mathrm{CI}$ : 40.0\%-55.0\%; $P=0.0336$ vs. ensifentrine alone; $n=3$; Fig. 11B), demonstrating that isoproterenol could potentiate the actions of ensifentrine. An even greater inhibition of GM-CSF production of $91.4 \% \pm 0.8 \%$ (95\% CI: $90.1 \%-92.5 \%$; $P=0.0000004$ vs. vehicle; $n=3$; Fig. $11 \mathrm{~B}$ ) was observed when ensifentrine was combined with a low concentration of dexamethasone which suggests additive mechanisms between the two drugs. Combining all three agonists did not further reduce IL- $1 \beta$-stimulated GM-CSF levels, presumably because ensifentrine and dexamethasone had already induced maximal downregulation (Fig. 11B). We also assessed GM-CSF mRNA expression after 8 hours challenge with IL- $1 \beta$. Under these conditions, ensifentrine reduced GM-CSF gene expression by $46.4 \% \pm 15 \%$ compared with cells treated with vehicle (95\% CI: $24.6 \%-68.0 \% ; P=0.0002 ; n=4$; Fig. $11 \mathrm{C}$ ), as we have previously shown in Fig. 6C. However, combining dexamethasone with ensifentrine and isoproterenol caused gene expression to be reduced to levels observed in nonchallenged cells. These findings are consistent with the protein levels and indicate that ensifentrine and dexamethasone act at the transcriptional level to downregulate GM-CSF production in response to inflammatory challenge.

Effect of Ensifentrine and Low-Concentration Dexamethasone Combination on GM-CSF Production by pHBE Cells. Encouraged by powerful anti-inflammatory effects of ensifentrine and dexamethasone in combination in CFBE410 ${ }^{-}$cells, we tested the combination in well differentiated pHBE cells. The protocol is shown in Fig. 12A. For these experiments, generally lower absolute levels of GM-CSF were measured compared with the experimental data presented in Fig. 9. This possibly reflects batch to batch variations in components of cell culture media; bovine serum albumin, for example, causes variable biologic effects between batches (Kane, 1983). Nevertheless, the fold changes in response to IL-1 $\beta$ remain comparable, indicating that the cultures responded to proinflammatory challenge in the same way with respect to GM-CSF production. Consistent with preceding experiments on $\mathrm{CFBE} 410^{-}$cells, the effect of each drug alone was not significant. However, in combination, ensifentrine and dexamethasone significantly reduced GM-CSF levels compared with vehicle control in non-CF pHBE cells $(P=0.0219 ; n=3$; Fig. 12$)$. Furthermore, the extent of the reduction in IL- $1 \beta$-stimulated GM-CSF production by ensifentrine + dexamethasone was significantly greater than by ensifentrine alone, demonstrating combining ensifentrine and low dose dexamethasone was an effective drug combination therapy to reduce proinflammatory mediators in airway epithelia.

Given that ensifentrine elevates intracellular cAMP and that cAMP can upregulate expression of the glucocorticoid receptor (GR) (Dong et al., 1989; Peñuelas et al., 1998), we tested if the effects observed when combining ensifentrine and dexamethasone might involve upregulation of GR expression. After 24 treatment with IL-1 $\beta$, GR mRNA expression was not altered by the presence of ensifentrine (Fig. 11, F and G). We 
A
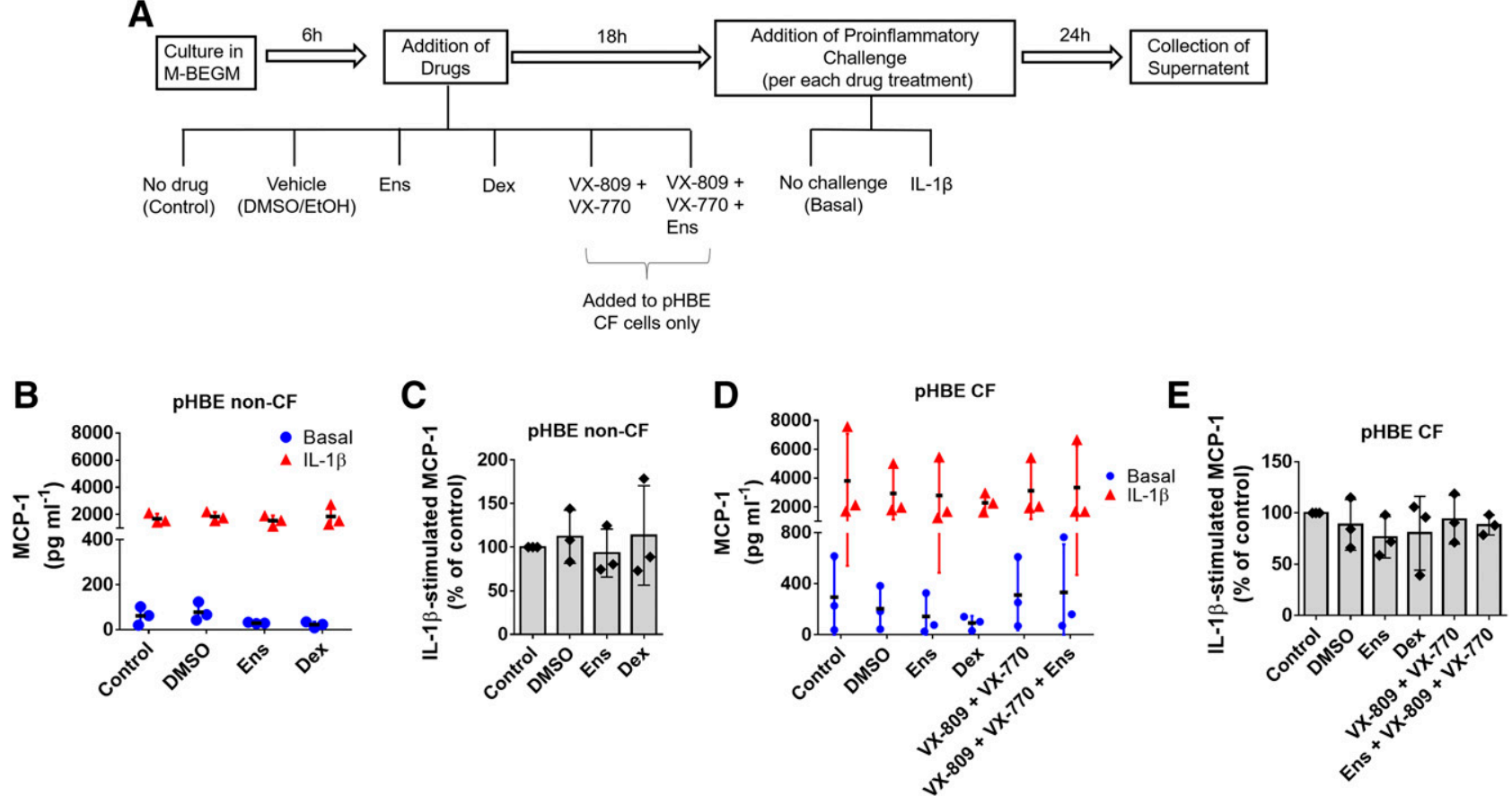

Fig. 9. Effects of ensifentrine on IL-1 $\beta$-stimulated production of MCP-1, GM-CSF, and IL-8 in well differentiated pHBE cells. (A) Experimental protocol for well differentiated $\mathrm{pHBE}$ cells cultured at the air-liquid interface for 28 days prior to experiments. Incubation in M-BEGM is followed by treatment with vehicle (DMSO; $0.05 \%$ ), ensifentrine (Ens; $10 \mu \mathrm{M}$ ) or dexamethasone (Dex; $5 \mathrm{nM}$ ) for 18 hours. Some CF pHBE cultures were also treated with VX$809(1 \mu \mathrm{M})+$ VX-770 $(100 \mathrm{nM})$, or VX-809 + VX-770 + ensifentrine. Cells were then challenged with the proinflammatory stimulus IL-1 $\beta(10 \mathrm{ng} / \mathrm{ml})$ for 24 hours. This was followed by supernatant collection, and cytokine quantification by MultiPlex ELISA. (B, F, and J) Summaries of absolute MCP-1, GM$\mathrm{CSF}$, and IL-8 levels in pHBE non-CF cells, respectively; these data are displayed as percent changes when normalized to IL-1 $\beta$-stimulated control cells in (C), (G), and (K). (D, H, and L) Summaries of absolute MCP-1, GM-CSF, and IL-8 levels in pHBE CF cells, respectively; these data are displayed as percent changes when normalized to IL-1 $\beta$-stimulated control cells in (E), (I), and (M). Individual data points and means \pm S.D. are shown; $N=3$.

therefore conclude that anti-inflammatory effects of ensifentrine in the presence of the low-concentration dexamethasone occur through some other mechanism.

\section{Discussion}

Novel therapeutic strategies are needed for the safe modulation of airway inflammation in CF. Suppression of the production of proinflammatory cytokines needs to not be to the extent where the immune response of the lung becomes compromised. The PDE inhibitor ensifentrine has previously been shown to reduce LPS-induced neutrophil and macrophage levels in the sputum of healthy patients, suggesting potential anti-inflammatory properties (Franciosi et al., 2013). In the present study, ensifentrine increased $[c A M P]_{i}$ in CFBE410- WT cells and stimulated robust, CFTRdependent secretion, demonstrating its ability to induce cAMP signaling in well differentiated human airway epithelia. These cells produced low levels of IL-10, IL-23 and IL-33 that were not modulated by 24 -hour proinflammatory challenge. In mice lungs, flagellin-induced increases in IL-33 are rapid and transient that, if similar in cultured human airway epithelia, would have been missed at the time points in our study (Wilson et al., 2012). The high levels of MCP-1, GM-CSF and IL-8 measured after 24 hours suggest these cytokines may be upregulated later or remain elevated for a longer time period than IL-33. This would be consistent with results from $P$. aeruginosa-infected mouse airways (Morris et al., 2009) and suggests that maintaining high levels of these cytokines may be required for late immune responses.
Basal levels of MCP-1, GM-CSF or IL-8 were not different between CFBE410 ${ }^{-}$WT and CFBE410 ${ }^{-}$F508del cells, nor between non-CF and $\mathrm{CF}$ pHBE cells implying that $\mathrm{CF}$ epithelia are not hyperinflammatory under basal conditions. This is consistent with a previous study demonstrating IL-6 and IL-8 are not elevated in CF pHBE cells (Becker et al., 2004). However, IL- $1 \beta$-induced elevations in MCP-1 and GMCSF levels were more pronounced in in CFBE410 ${ }^{-}$F508del cells compared with CFBE410 ${ }^{-}$WT cells and GM-CSF levels were significantly higher in IL- $1 \beta$-challenged CF pHBE cells compared with non-CF pHBE cells. IL-8 levels did not show these differences, indicating that $\mathrm{CF}$ epithelia are hyperinflammatory when challenged with proinflammatory stimuli but only with respect to specific cytokines. In well differentiated CFBE410 ${ }^{-}$F508del cells, ensifentrine reduced IL-1 $\beta-$ induced MCP-1 and GM-CSF production to that of those measured in control, IL- $1 \beta$-challenged CFBE $410^{-}$WT cells, suggesting that ensifentrine can dampen the heightened response to proinflammatory stimuli in $\mathrm{CF}$ airway epithelia. The effects of ensifentrine were mediated exclusively through PDE4 inhibition, even though ensifentrine exhibits greater potency for PDE3 versus PDE4 (Boswell-Smith et al., 2006). These findings are consistent with our previous studies that detected little PDE3A expression in well differentiated airway epithelia and showed that ensifentrine-stimulated CFTR activity is identical to stimulation by selective PDE4 inhibitors (Turner et al., 2016). Furthermore, the anti-inflammatory effects of ensifentrine are comparable to those of alternative PDE4 inhibitors reported in other models of lung inflammation (Profita et al., 2003; Sasaki and Manabe, 2004; Buenestado 

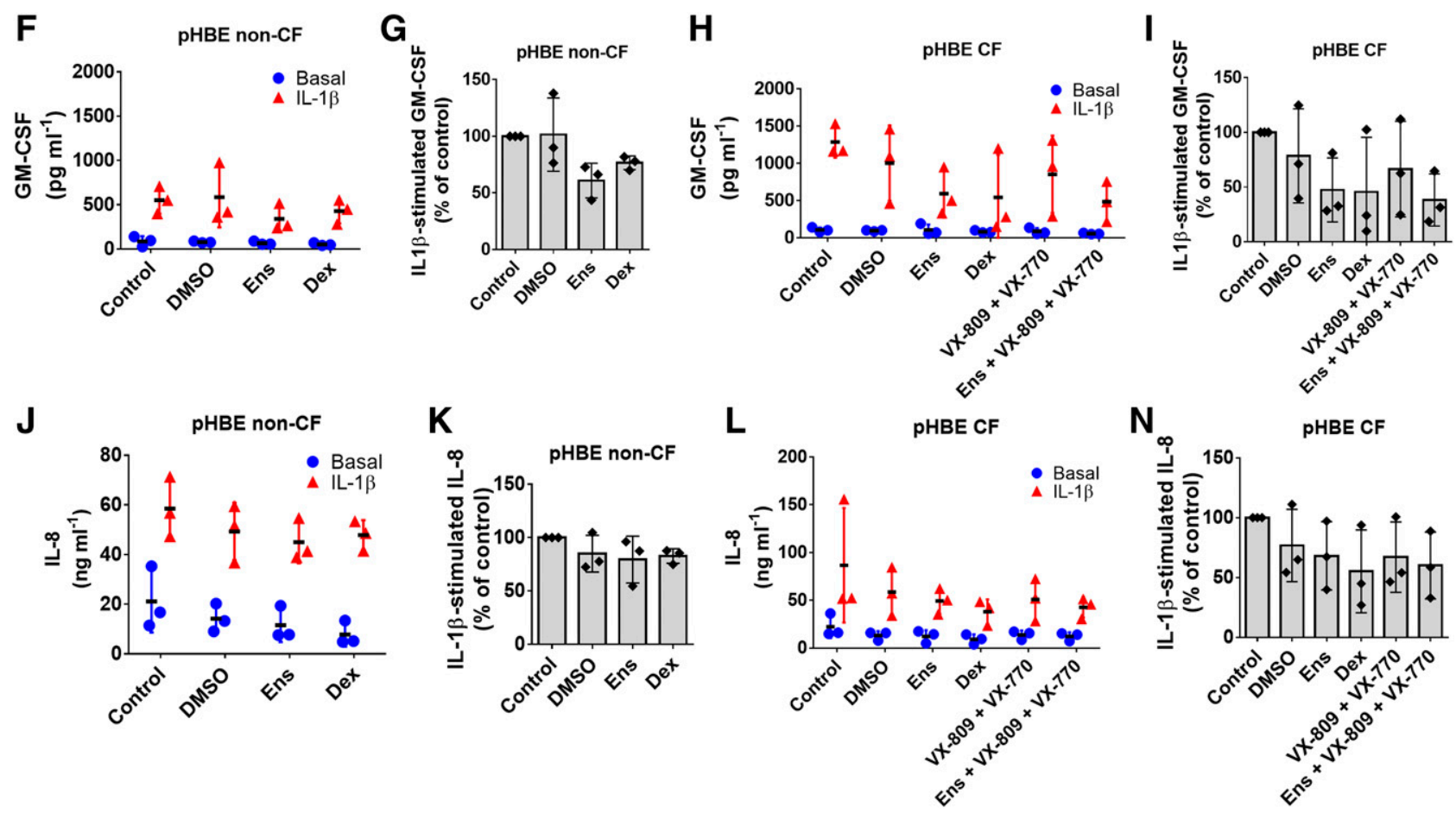

Fig. 9. Continued.

et al., 2012, 2013). Surprisingly, cytokine production in CFBE410 ${ }^{-}$WT cells was modulated by ethanol which may be a consequence of previously described ethanol-induced upregulation of immune receptors (Bailey et al., 2009).

In well differentiated $\mathrm{pHBE}$ cells from non-CF and $\mathrm{CF}$ donors, we observed ensifentrine to not significantly alter MCP-1, GM-CSF or IL-8 under basal or inflammatory conditions. However, there was a trend for ensifentrine to reduce
IL-1 $\beta$-stimulated GM-CSF production in CF pHBE cells to levels seen in control, IL- $1 \beta$-stimulated non-CF pHBE cells. These observations support data from CFBE $410^{-}$cells and suggests that ensifentrine can diminish the hyperinflammation in response to proinflammatory stimuli observed in $\mathrm{CF}$ airway epithelia. Levels of proinflammatory mediators were not affected by partial correction of F508del CFTR using VX-809+ VX-770. These results were unexpected as VX-809 + VX-770

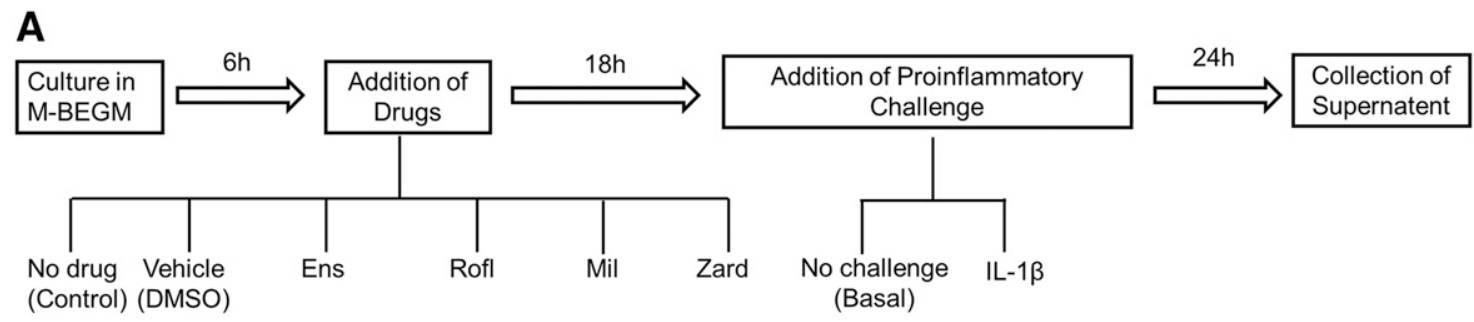

B

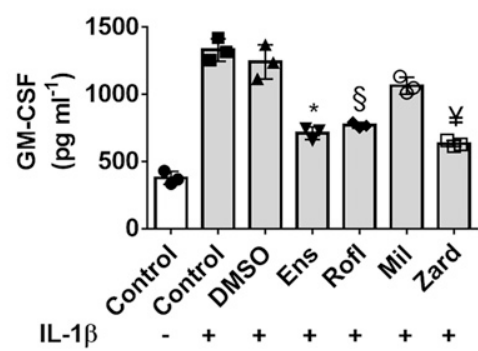

C

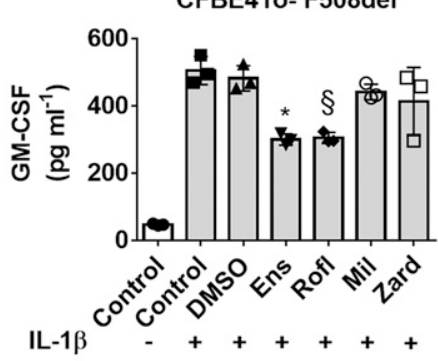

Fig. 10. Effects of other PDE3 and PDE4 inhibitors on stimulated GM-CSF production in CFBE41o ${ }^{-}$cells. (A) Schematic of protocol for treating CFBE410 ${ }^{-}$cells cultured at the air-liquid interface for 7 days. Incubation in M-BEGM was followed by treatment with vehicle (DMSO; $0.05 \%$ ), ensifentrine (Ens; $10 \mu \mathrm{M}$ ), roflumilast (Rofl; $1 \mu \mathrm{M}$ ), milrinone (Mil; $10 \mu \mathrm{M}$ ), and zardaverine (Zard; $10 \mu \mathrm{M})$ for 18 hours. Cells were then challenged with IL-1 $\beta(10 \mathrm{ng} / \mathrm{ml})$ before the supernatants were collected, and GM-CSF levels were quantified by SinglePlex ELISA. (B) Summary of GM-CSF levels in CFBE41o- WT cells. $* P=0.0025 ;{ }^{\S} P=0.0032 ;{ }^{¥} P=0.0012$ in unpaired $t$ tests vs. vehicle control. (C) Summary of GM-CSF levels in CFBE41o ${ }^{-}$F508del cells. ${ }^{*} P=0.0016 ;{ }^{\S} P=0.0017$ in unpaired $t$ tests vs. vehicle control. Individual data points and means \pm S.D. are shown; $N=3$. 

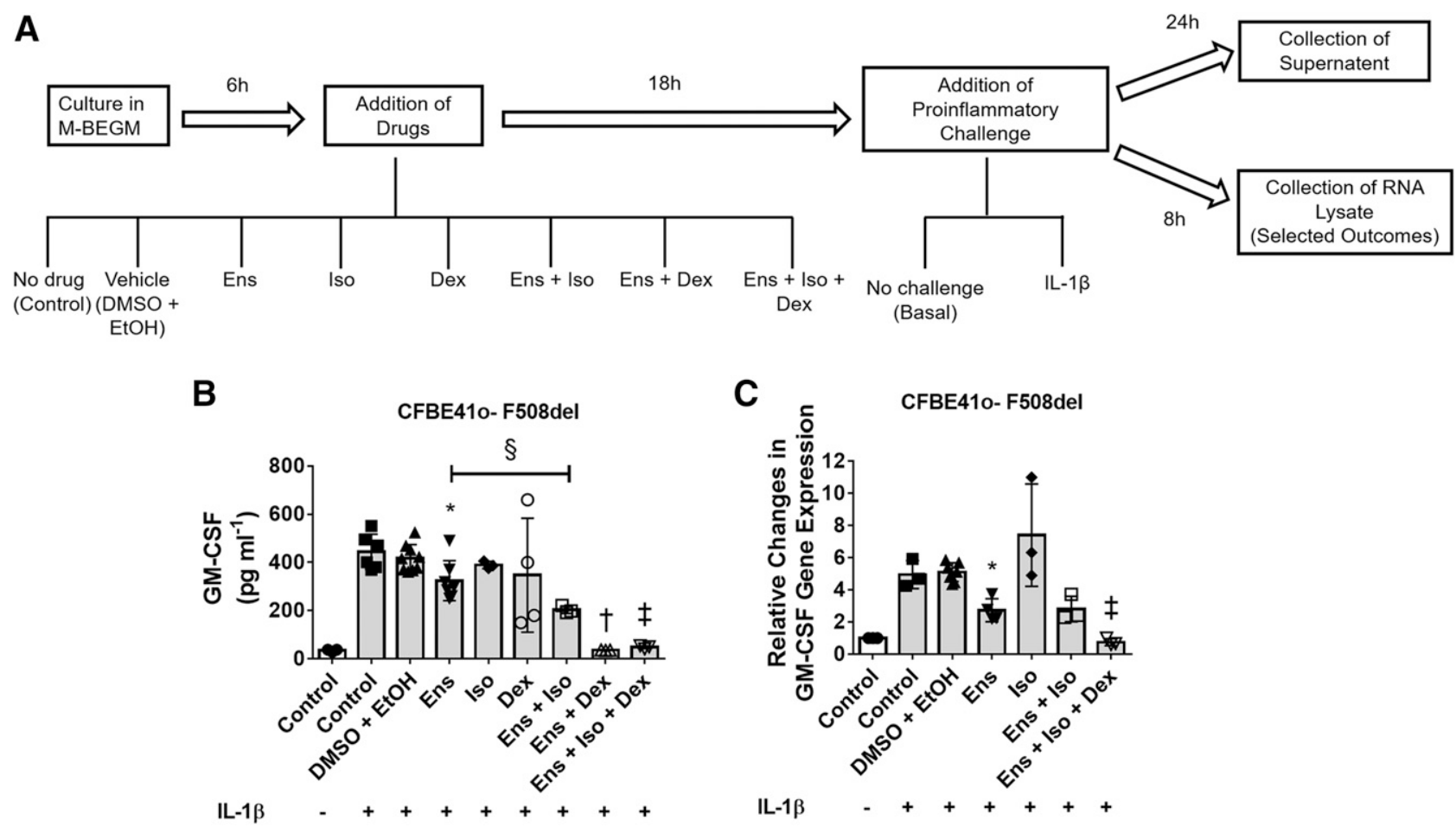

Fig. 11. Effects of combining ensifentrine with isoproterenol and low-concentration dexamethasone on stimulated GM-CSF production in CFBE41o F508del cells. (A) Schematic of protocol for treating CFBE41o- cells cultured at the air-liquid interface for 7 days. Incubation in M-BEGM was followed by treatment with vehicle (DMSO; 0.05\% + ethanol; 0.05\%), ensifentrine (Ens; $10 \mu \mathrm{M}$ ), isoproterenol (Iso; $10 \mathrm{nM}$ ), dexamethasone (Dex; $5 \mathrm{nM}$ ), ensifentrine + isoproterenol, ensifentrine + dexamethasone, and ensifentrine + isoproterenol + dexamethasone for 18 hours. Cells were then challenged with IL-1 $\beta(10 \mathrm{ng} / \mathrm{ml})$ for either 8 hours or 24 hours before the RNA lysate or supernatants were collected, GM-CSF mRNA levels were quantified by qPCR and protein levels were quantified by SinglePlex ELISA. Note that RNA was only collected for selected outcomes. (B) Summary of GM-CSF levels in CFBE410 ${ }^{-}$F508del cells. ${ }^{*} P=0.017$ in unpaired $t$ test vs. vehicle control; ${ }^{\circledR} P=0.0427$ in unpaired $t$ test vs. ensifentrine alone; ${ }^{\dagger} P=0.00000044$ in unpaired $t$ test vs. vehicle control; ${ }^{\ddagger} P=0.00000061$ in unpaired $t$ test vs. vehicle control. (C) Summary of GM-CSF gene expression levels in CFBE410 ${ }^{-}$ F508del cells. $* * P=0.002$ in unpaired $t$ test vs. vehicle control; ${ }^{\ddagger} P=0.000002$ in unpaired $t$ test vs. vehicle control. Individual data points and means \pm S.D. are shown; $N=3$ to 4 .

have been shown to reduce IL-8 mRNA levels in well differentiated $\mathrm{CF}$ pHBE cells exposed to $P$. aeruginosa exoproducts (Ruffin et al., 2018). The different results in the two studies could be due to the use of different proinflammatory stimuli and/or exposure conditions.

Although ensifentrine and a low concentration of dexamethasone had only small effects on GM-CSF production when used separately, in combination we observed a significant downregulation of GM-CSF. The effects were mirrored in well differentiated pHBE cells, indicating that using drugs in combination has more potent anti-inflammatory effects than either drug alone. PDE4 inhibition has previously been shown to enhance corticosteroid-induced activation of the glucocorticoid response element in BEAS-2B and pHBE cells and potentiate dexamethasone-induced downregulation of IL-8 and $\mathrm{TNF} \alpha$ in pHBE cells from patients with COPD (Moodley et al., 2013; BinMahfouz et al., 2015; Reddy et al., 2020). Furthermore, in human airway epithelia, TNF- $\alpha$-stimulated GM-CSF production is significantly reduced in cells treated with both the glucocorticoid budesonide and the cAMPelevating formoterol (Korn et al., 2001) with these effects of budesonide and formoterol further enhanced when MRP4dependent cAMP efflux is also blocked (Huff et al., 2018). These data are in good agreement with our findings and imply combination therapies of glucocorticoids and cAMP-elevating agents are an effective approach to modulate airway inflammation. The mechanism of the interaction between corticosteroids and cAMP-elevating agonists remains uncertain (Taylor and Hancox, 2000). In neurons, synergism between forskolin and dexamethasone has been ascribed to cAMP upregulation of GR expression (Scarcériaux et al., 1995), whereas roflumilast enhances the expression of GR $\alpha$ in COPD pHBE cells after 6-hour treatment (Reddy et al., 2020). However, we did not observe an effect of ensifentrine on GR mRNA expression, perhaps due to our analysis of GR expression occurring later (24 hours) than in previous studies. Alternatively, ensifentrine may increase GR efficiency, e.g., by altering its intracellular localization. Dexamethasone reduces proinflammatory cytokine production in CF airway epithelia in vitro (Rebeyrol et al., 2012), however the concentrations of dexamethasone used generally exceed the clinical dose. The glucocorticoid prednisone has been studied in clinical trials for CF. Although it slowed the progression of lung disease at high doses, adverse effects were observed (Eigen et al., 1995; Cheng et al., 2015). Our results suggest that it may be possible to reduce inflammation in CF airways and avoid harmful side effects of glucocorticoids by combining a low dose of prednisone with a PDE4 inhibitor (i.e., ensifentrine). Recently, preliminary investigations into the use of dexamethasone as a treatment of Covid-19 has suggested potential benefits (Horby et al., 2020). Therefore, combining dexamethasone with PDE4 inhibitors would be interesting to examine in these patients.

Isoproterenol was also able to enhance the effect of ensifentrine on IL- $1 \beta$-stimulated GM-CSF production. PDE4 inhibitors 

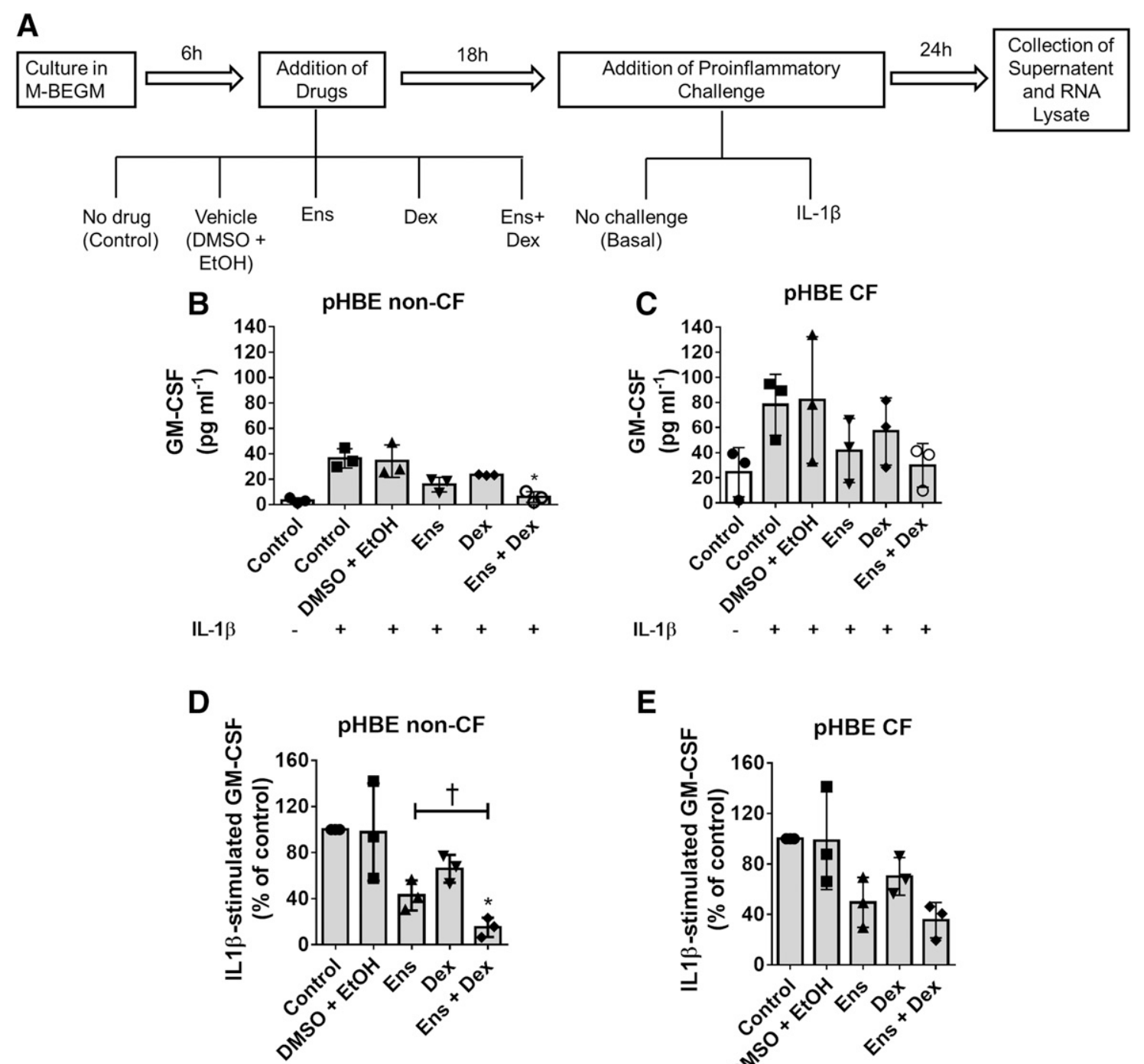

$\mathbf{E}$

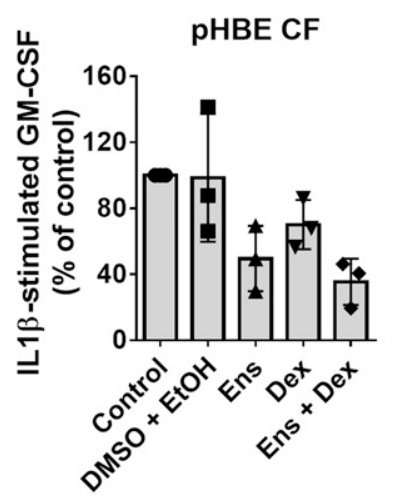

$\mathbf{F}$

PHBE non-CF

G
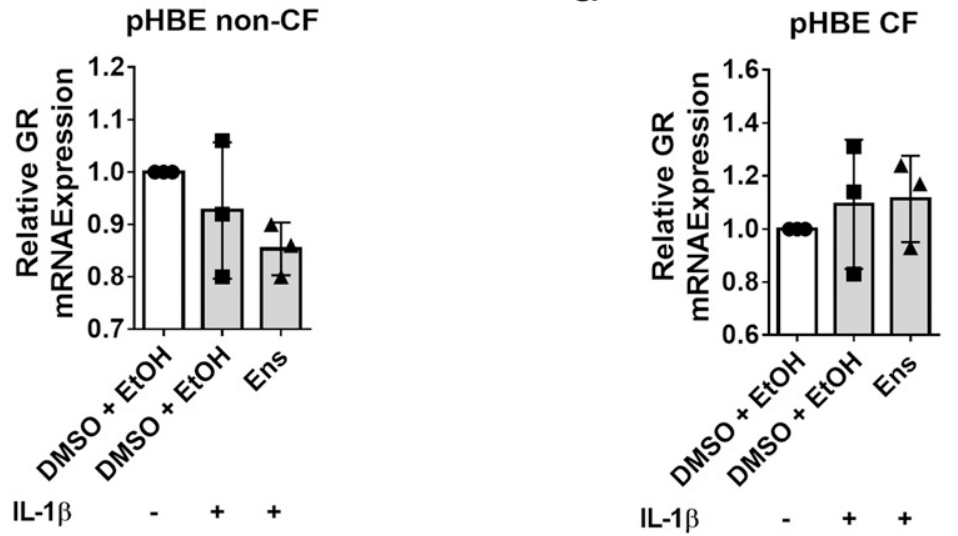

Fig. 12. Effects of combining ensifentrine and low-concentration dexamethasone on GM-CSF production in well differentiated pHBE cells. (A) Experimental protocol for well differentiated pHBE cells cultured at the air-liquid interface for 28 days prior to experiments. Incubation in M-BEGM is followed by treatment with vehicle (DMSO; 0.05\% + ethanol; 0.05\%), ensifentrine (Ens; $10 \mu \mathrm{M})$, dexamethasone (Dex; $5 \mathrm{nM})$, or ensifentrine + dexamethasone for 18 hours. Cells were then challenged with the proinflammatory stimulus IL-1 $\beta$ ( $10 \mathrm{ng} / \mathrm{ml}$ ) for 24 hours. This was followed by supernatant collection for GM-CSF level quantification by MultiPlex ELISA, and RNA collection for assessment of GR mRNA expression. (B and C) Summaries of absolute GM-CSF levels in, respectively, non-CF and CF pHBE cells. ${ }^{*} P=0.0219$ in unpaired $t$ test vs. vehicle. (D and E) GM-CSF levels presented as \% of IL-1 $\beta$-challenged and untreated cells $\left(=\right.$ control). $* P=0.0294$ in unpaired $t$ test vs. vehicle; ${ }^{\dagger} P=0.0362$ in unpaired $t$ test vs. ensifentrine alone. Individual data points and means \pm S.D. are shown, $N=3$. ( $\mathrm{F}$ and G) GR mRNA expression in, respectively, non-CF and CF pHBE cells. Individual data points and means \pm S.D. are shown; $N=3$. 
can enhance the expression of genes under control of the CRE in BEAS-2B cells and enhance the sensitivity of CREdependent gene expression to $\beta_{2}$ receptor agonists. Interestingly, this effect is most prominent when both PDE3 and PDE4 inhibition is achieved (BinMahfouz et al., 2015). Although their effectiveness is disputed, long acting, inhaled bronchodilators, including $\beta_{2}$ agonists, are prescribed for patients with CF (Smith and Edwards, 2017). Ensifentrine is also clinically administered by inhalation and thus could be combined with inhalation of $\beta_{2}$ agonists, either simultaneously or sequentially, to enhance the antiinflammatory effects of ensifentrine.

Elevated $\left[\mathrm{Cl}^{-}\right]_{\mathrm{i}}$ in $\mathrm{CF}$ bronchial epithelia has been attributed to contributing to hyperinflammation in CF airways in which $\left[\mathrm{Cl}^{-}\right]_{i}$ per se can regulate the expression of proinflammatory cytokines (Valdivieso et al., 2016; Clauzure et al., 2017). As a result, stimulation of $\mathrm{Cl}^{-}$secretion through CFTR has been proposed to dampen inflammation in CF airway epithelia (Perez et al., 2007; Zhang et al., 2018). However, although ensifentrine stimulated CFTR activity in CFBE41oWT cells, ensifentrine and forskolin elicited their antiinflammatory effects in CFBE410 ${ }^{-}$F508del cells, in which cAMP agonists induce negligible $\mathrm{Cl}^{-}$secretion (Bebok et al., 2005). Therefore, these data indicate that elevated $[\mathrm{cAMP}]_{\mathrm{i}}$ per se mediates the anti-inflammatory effects and not the downstream activation of CFTR. The lack of an effect of VX$809+\mathrm{VX}-770$ is also consistent with CFTR activity being independent of proinflammatory cytokine production. Instead, cAMP-dependent regulation of $\mathrm{NF} \kappa \mathrm{B}$ signaling and/or regulation of genes regulated by the cAMP-sensitive transcription factor CREB (Rovin et al., 1995; Koga et al., 2016) are more likely to underlie the effects of ensifentrine.

Although in bronchial epithelial cells ensifentrine elicited similar anti-inflammatory effects to selective PDE4 inhibitors, CF pathology and inflammation also involve other cell types that express PDE3. For instance, recent reports have indicated that CFTR dysfunction in platelets contribute to the excessive inflammation in CF airways (Ortiz-Muñoz et al., 2020). PDE3 inhibition can modulate platelet function (Gresele et al., 2011); therefore, PDE3 inhibitors may exert antiinflammatory effects in CF platelets rather than directly on bronchial epithelium. Furthermore, our model of cultured bronchial epithelial cells lacks the surrounding tissue, notably submucosal glands that secrete the majority of the airway surface liquid and airway smooth muscle. Unpublished observations from our laboratory show that ensifentrine stimulates CFTR-dependent fluid and $\mathrm{HCO}_{3}^{-}$secretion from Calu-3 cells, a cell line often used as a model for submucosal gland serous cells (Shen et al., 1994) through inhibition of PDE3 rather than PDE4, whereas PDE3 inhibitors, including ensifentrine, induce significant bronchodilation in COPD (Page, 2014). Thus, dual PDE3/4 inhibitors are attractive anti-CF therapeutics given their ability to inhibit PDEs expressed in different cell types to elicit multiple beneficial effects across a range of tissue involved in CF pathology. Given the broad range of tissues that ensifentrine can modulate, administration by inhalation enables it to be restricted to the airways and avoid potential adverse effects in other nonairway tissues.

In summary, we have demonstrated that ensifentrine both stimulates CFTR and beneficially modulates the release of proinflammatory factors in well differentiated bronchial epithelial cells, particularly when combined with low concentrations of $\beta_{2}$-adrenergic or corticosteroids. Although the precise molecular mechanisms of ensifentrine-inhibited cytokine production remain to be elucidated, the present study is the first to examine PDE inhibitor effects on inflammatory responses in well differentiated human bronchial epithelia that express F508del CFTR. Given its ability to stimulate mutant CFTR and reduce airway epithelial inflammatory responses, our findings encourage further clinical testing of ensifentrine as a CF therapeutic.

\section{Authorship Contributions}

Participated in research design: Turner, Dauletbaev, Lands,

Hanrahan.

Conducted experiments: Turner.

Performed data analysis: Turner, Dauletbaev.

Wrote or contributed to the writing of the manuscript: Turner, Dauletbaev, Lands, Hanrahan.

\section{References}

Abbott-Banner KH and Page CP (2014) Dual PDE3/4 and PDE4 inhibitors: novel treatments for COPD and other inflammatory airway diseases. Basic Clin Pharmacol Toxicol 114:365-376.

Al-Nakkash L and Hwang TC (1999) Activation of wild-type and deltaF508-CFTR by phosphodiesterase inhibitors through cAMP-dependent and -independent mechanisms. Pflugers Arch 437:553-561.

Bailey KL, Wyatt TA, Romberger DJ, and Sisson JH (2009) Alcohol functionally upregulates Toll-like receptor 2 in airway epithelial cells. Alcohol Clin Exp Res 33: 499-504.

Balloy V, Thévenot G, Bienvenu T, Morand P, Corvol H, Clement A, Ramphal R, Hubert D, and Chignard M (2014) Flagellin concentrations in expectorations from cystic fibrosis patients. BMC Pulm Med 14:100.

Banner KH and Page CP (1995) Theophylline and selective phosphodiesterase inhibitors as anti-inflammatory drugs in the treatment of bronchial asthma. Eur Respir J 8:996-1000.

Barnes AP, Livera G, Huang P, Sun C, O’Neal WK, Conti M, Stutts MJ, and Milgram SL (2005) Phosphodiesterase 4D forms a cAMP diffusion barrier at the apical membrane of the airway epithelium. J Biol Chem 280:7997-8003.

Bebok Z, Collawn JF, Wakefield J, Parker W, Li Y, Varga K, Sorscher EJ, and Clancy JP (2005) Failure of cAMP agonists to activate rescued deltaF508 CFTR in CFBE41o- airway epithelial monolayers. J Physiol 569:601-615.

Becher B, Tugues S, and Greter M (2016) GM-CSF: from growth factor to central mediator of tissue inflammation. Immunity 45:963-973.

Becker MN, Sauer MS, Muhlebach MS, Hirsh AJ, Wu Q, Verghese MW, and Randell $\mathrm{SH}$ (2004) Cytokine secretion by cystic fibrosis airway epithelial cells. Am J Respir Crit Care Med 169:645-653.

BinMahfouz H, Borthakur B, Yan D, George T, Giembycz MA, and Newton R (2015) Superiority of combined phosphodiesterase PDE3/PDE4 inhibition over PDE4 inhibition alone on glucocorticoid- and long-acting $\beta 2$-adrenoceptor agonist-induced gene expression in human airway epithelial cells. Mol Pharmacol 87:64-76.

Blanchard E, Zlock L, Lao A, Mika D, Namkung W, Xie M, Scheitrum C, Gruenert DC, Verkman AS, Finkbeiner WE, et al. (2014) Anchored PDE4 regulates chloride conductance in wild-type and $\triangle$ F508-CFTR human airway epithelia. FASEB J 28: 791-801.

Bonfield TL, Konstan MW, Burfeind P, Panuska JR, Hilliard JB, and Berger M (1995a) Normal bronchial epithelial cells constitutively produce the antiinflammatory cytokine interleukin-10, which is downregulated in cystic fibrosis. Am J Respir Cell Mol Biol 13:257-261.

Bonfield TL, Panuska JR, Konstan MW, Hilliard KA, Hilliard JB, Ghnaim H, and Berger M (1995b) Inflammatory cytokines in cystic fibrosis lungs. Am J Respir Crit Care Med 152:2111-2118.

Boswell-Smith V, Spina D, Oxford AW, Comer MB, Seeds EA, and Page CP (2006) The pharmacology of two novel long-acting phosphodiesterase $3 / 4$ inhibitors, RPL554 [9,10-dimethoxy-2(2,4,6-trimethylphenylimino)-3-(n-carbamoyl-2-aminoethyl)-3,4,6,7-tetrahydro-2H-pyrimido[6,1-a]isoquinolin-4-one] and RPL565 [6,7dihydro-2-(2,6-diisopropylphenoxy)-9,10-dimethoxy-4H-pyrimido[6,1-a]isoquinolin4-one]. J Pharmacol Exp Ther 318:840-848.

Brennan S, Sly PD, Gangell CL, Sturges N, Winfield K, Wikstrom M, Gard S, and Upham JW; AREST CF (2009) Alveolar macrophages and CC chemokines are increased in children with cystic fibrosis. Eur Respir $J$ 34:655-661.

Buenestado A, Chaumais MC, Grassin-Delyle S, Risse PA, Naline E, Longchampt E, Tenor H, and Devillier P (2013) Roflumilast inhibits lipopolysaccharide-induced tumor necrosis factor- $\alpha$ and chemokine production by human lung parenchyma. PLoS One 8:e74640.

Buenestado A, Grassin-Delyle S, Guitard F, Naline E, Faisy C, Israël-Biet D, Sage E, Bellamy JF, Tenor H, and Devillier P (2012) Roflumilast inhibits the release of chemokines and TNF- $\alpha$ from human lung macrophages stimulated with lipopolysaccharide. Br J Pharmacol 165:1877-1890.

Cantin AM, Hartl D, Konstan MW, and Chmiel JF (2015) Inflammation in cystic fibrosis lung disease: pathogenesis and therapy. J Cyst Fibros 14:419-430.

Cheng K, Ashby D, and Smyth RL (2015) Oral steroids for long-term use in cystic fibrosis. Cochrane Database Syst Rev 2015:CD000407.

Clauzure M, Valdivieso AG, Massip-Copiz MM, Mori C, Dugour AV, Figueroa JM, and Santa-Coloma TA (2017) Intracellular chloride concentration changes 
modulate IL-1 $\beta$ expression and secretion in human bronchial epithelial cultured cells. J Cell Biochem 118:2131-2140.

Cobb BR, Fan L, Kovacs TE, Sorscher EJ, and Clancy JP (2003) Adenosine receptors and phosphodiesterase inhibitors stimulate Cl- secretion in Calu-3 cells. Am $J$ Respir Cell Mol Biol 29:410-418.

Cox G, Ohtoshi T, Vancheri C, Denburg JA, Dolovich J, Gauldie J, and Jordana M (1991) Promotion of eosinophil survival by human bronchial epithelial cells and its modulation by steroids. Am J Respir Cell Mol Biol 4:525-531.

Dauletbaev N, Lam J, Eklove D, Iskandar M, and Lands LC (2010) Ibuprofen modulates NF-kB activity but not IL-8 production in cystic fibrosis respiratory epithelial cells. Respiration 79:234-242.

Dean TP, Dai Y, Shute JK, Church MK, and Warner JO (1993) Interleukin-8 concentrations are elevated in bronchoalveolar lavage, sputum, and sera of children with cystic fibrosis. Pediatr Res 34:159-161.

Deshmane SL, Kremlev S, Amini S, and Sawaya BE (2009) Monocyte chemoattractant protein-1 (MCP-1): an overview. J Interferon Cytokine Res 29:313-326.

Dong Y, Aronsson M, Gustafsson JA, and Okret S (1989) The mechanism of cAMP induced glucocorticoid receptor expression. Correlation to cellular glucocorticoid response. J Biol Chem 264:13679-13683.

Drake LY and Kita H (2017) IL-33: biological properties, functions, and roles in airway disease. Immunol Rev 278:173-184.

Eckrich J, Zissler UM, Serve F, Leutz P, Smaczny C, Schmitt-Grohé S, Fussbroich D, Schubert R, Zielen S, and Eickmeier O (2017) Airway inflammation in mild cystic fibrosis. J Cyst Fibros 16:107-115.

Eickmeier O, Huebner M, Herrmann E, Zissler U, Rosewich M, Baer PC, Buhl R, Schmitt-Grohé S, Zielen S, and Schubert R (2010) Sputum biomarker profiles in cystic fibrosis (CF) and chronic obstructive pulmonary disease (COPD) and association between pulmonary function. Cytokine 50:152-157.

Eigen H, Rosenstein BJ, FitzSimmons S, and Schidlow DV; Cystic Fibrosis Foundation Prednisone Trial Group (1995) A multicenter study of alternate-day prednisone therapy in patients with cystic fibrosis. J Pediatr 126:515-523.

Elborn JS (2016) Cystic fibrosis. Lancet 388:2519-2531.

Franciosi LG, Diamant Z, Banner KH, Zuiker R, Morelli N, Kamerling IM, de Kam ML, Burggraaf J, Cohen AF, Cazzola M, et al. (2013) Efficacy and safety of RPL554, a dual PDE3 and PDE4 inhibitor, in healthy volunteers and in patients with asthma or chronic obstructive pulmonary disease: findings from four clinical trials. Lancet Respir Med 1:714-727.

Fulcher ML, Gabriel S, Burns KA, Yankaskas JR, and Randell SH (2005) Welldifferentiated human airway epithelial cell cultures. Methods $\mathrm{Mol}$ Med 107: 183-206.

Gresele P, Momi S, and Falcinelli E (2011) Anti-platelet therapy: phosphodiesterase inhibitors. Br J Clin Pharmacol 72:634-646.

Horby P, Lim WS, Emberson JR, Mafham M, Bell JL, Linsell L, Staplin N, Brightling C, Ustianowski A, Elmahi E, et al.; RECOVERY Collaborative Group (2020) Dexamethasone in hospitalized patients with covid-19 - preliminary report. $N$ Engl $J$ Med DOI: 10.1056/NEJMoa2021436 [published ahead of print].

Huff RD, Rider CF, Yan D, Newton R, Giembycz MA, Carlsten C, and Hirota JA (2018) Inhibition of ABCC4 potentiates combination beta agonist and glucocorticoid responses in human airway epithelial cells. J Allergy Clin Immunol 141: 1127-1130.e5

Hybiske K, Ichikawa JK, Huang V, Lory SJ, and Machen TE (2004) Cystic fibrosis airway epithelial cell polarity and bacterial flagellin determine host response to Pseudomonas aeruginosa. Cell Microbiol 6:49-63.

Jensen PO, Moser C, Kharazmi A, Presler T, Koch C, and Høiby N (2006) Increased serum concentration of G-CSF in cystic fibrosis patients with chronic Pseudomonas aeruginosa pneumonia. J Cyst Fibros 5:145-151.

Kane MT (1983) Variability in different lots of commercial bovine serum albumin affects cell multiplication and hatching of rabbit blastocysts in culture. $J$ Reprod Fertil 69:555-558.

Koga Y, Hisada T, Ishizuka T, Utsugi M, Ono A, Yatomi M, Kamide Y, Aoki-Saito H Tsurumaki $\mathrm{H}$, Dobashi K, et al. (2016) CREB regulates TNF- $\alpha$-induced GM-CSF secretion via p38 MAPK in human lung fibroblasts. Allergol Int 65:406-413.

Korn SH, Jerre A, and Brattsand R (2001) Effects of formoterol and budesonide on GM-CSF and IL-8 secretion by triggered human bronchial epithelial cells. Eur Respir J 17:1070-1077.

Lands LC and Stanojevic S (2019) Oral non-steroidal anti-inflammatory drug therapy for lung disease in cystic fibrosis. Cochrane Database Syst Rev 9:CD001505.

Lee HS, Park DE, Lee JW, Chang Y, Kim HY, Song WJ, Kang HR, Park HW, Chang YS, and Cho SH (2017) IL-23 secreted by bronchial epithelial cells contributes to allergic sensitization in asthma model: role of IL-23 secreted by bronchial epithelial cells. Am J Physiol Lung Cell Mol Physiol 312:L13-L21.

Liu S, Veilleux A, Zhang L, Young A, Kwok E, Laliberté F, Chung C, Tota MR, Dubé D, Friesen RW, et al. (2005) Dynamic activation of cystic fibrosis transmembrane conductance regulator by type 3 and type $4 \mathrm{D}$ phosphodiesterase inhibitors. $J$ Pharmacol Exp Ther 314:846-854.

Lundien MC, Mohammed KA, Nasreen N, Tepper RS, Hardwick JA, Sanders KL, Van Horn RD, and Antony VB (2002) Induction of MCP-1 expression in airway epithelial cells: role of CCR2 receptor in airway epithelial injury. J Clin Immunol 22:144-152.

Mathew JS and Sharma RP (2000) Effect of all-trans-retinoic acid on cytokine production in a murine macrophage cell line. Int $J$ Immunopharmacol 22 693-706

Maurice DH, Ke H, Ahmad F, Wang Y, Chung J, and Manganiello VC (2014) Advances in targeting cyclic nucleotide phosphodiesterases. Nat Rev Drug Discov 13:290-314.

Moodley T, Wilson SM, Joshi T, Rider CF, Sharma P, Yan D, Newton R, and Giembycz MA (2013) Phosphodiesterase 4 inhibitors augment the ability of formoterol to enhance glucocorticoid-dependent gene transcription in human airway epithelial cells: a novel mechanism for the clinical efficacy of roflumilast in severe chronic obstructive pulmonary disease. Mol Pharmacol 83:894-906.
Morris AE, Liggitt HD, Hawn TR, and Skerrett SJ (2009) Role of Toll-like receptor 5 in the innate immune response to acute P. aeruginosa pneumonia. Am J Physio Lung Cell Mol Physiol 297:L1112-L1119.

Moussion C, Ortega N, and Girard JP (2008) The IL-1-like cytokine IL-33 is constitutively expressed in the nucleus of endothelial cells and epithelial cells in vivo: a novel 'alarmin'? PLoS One 3:e3331.

Mukaida N (2003) Pathophysiological roles of interleukin-8/CXCL8 in pulmonary diseases. Am J Physiol Lung Cell Mol Physiol 284:L566-L577.

Myou S, Fujimura M, Kamio Y, Ishiura Y, Tachibana H, Hirose T, Hashimoto T, and Matsuda T (1999) Bronchodilator effect of inhaled olprinone, a phosphodiesterase 3 inhibitor, in asthmatic patients. Am J Respir Crit Care Med 160:817-820. Ortiz-Muñoz G, Yu MA, Lefrançais E, Mallavia B, Valet C, Tian JJ, Ranucci S, Wang KM, Liu Z, Kwaan N, et al. (2020) Cystic fibrosis transmembrane conductance regulator dysfunction in platelets drives lung hyperinflammation. J Clin Invest 130:2041-2053.

Osika E, Cavaillon JM, Chadelat K, Boule M, Fitting C, Tournier G, and Clement A (1999) Distinct sputum cytokine profiles in cystic fibrosis and other chronic inflammatory airway disease. Eur Respir $J$ 14:339-346.

Page CP (2014) Phosphodiesterase inhibitors for the treatment of asthma and chronic obstructive pulmonary disease. Int Arch Allergy Immunol 165:152-164.

Peñuelas I, Encío IJ, López-Moratalla N, and Santiago E (1998) cAMP activates transcription of the human glucocorticoid receptor gene promoter. J Steroid Biochem Mol Biol 67:89-94.

Perez A, Issler AC, Cotton CU, Kelley TJ, Verkman AS, and Davis PB (2007) CFTR inhibition mimics the cystic fibrosis inflammatory profile. Am J Physiol Lung Cell Mol Physiol 292:L383-L395.

Préfontaine D, Nadigel J, Chouiali F, Audusseau S, Semlali A, Chakir J, Martin JG, and Hamid Q (2010) Increased IL-33 expression by epithelial cells in bronchial asthma. J Allergy Clin Immunol 125:752-754.

Principi N, Blasi F, and Esposito S (2015) Azithromycin use in patients with cystic fibrosis. Eur J Clin Microbiol Infect Dis 34:1071-1079.

Profita M, Chiappara G, Mirabella F, Di Giorgi R, Chimenti L, Costanzo G, Riccobono L, Bellia V, Bousquet J, and Vignola AM (2003) Effect of cilomilast (Ariflo) on TNFalpha, IL-8, and GM-CSF release by airway cells of patients with COPD. Thorax 58:573-579.

Rao SP, Hayashi T, and Catanzaro A (2000) Release of monocyte chemoattractant protein (MCP)-1 by a human alveolar epithelial cell line in response to mycobacterium avium. FEMS Immunol Med Microbiol 29:1-7.

Ratjen F, Saiman L, Mayer-Hamblett N, Lands LC, Kloster M, Thompson V, Emmett P, Marshall B, Accurso F, Sagel S, et al. (2012) Effect of azithromycin on systemic markers of inflammation in patients with cystic fibrosis uninfected with Pseudomonas aeruginosa. Chest 142:1259-1266.

Rebeyrol C, Saint-Criq V, Guillot L, Riffault L, Corvol H, Chadelat K, Ray DW, Clement A, Tabary O, and Le Rouzic P (2012) Glucocorticoids reduce inflammation in cystic fibrosis bronchial epithelial cells. Cell Signal 24:1093-1099.

Reddy AT, Lakshmi SP, Banno A, and Reddy RC (2020) Glucocorticoid receptor $\alpha$ mediates roflumilast's ability to restore dexamethasone sensitivity in COPD. Int $J$ Chron Obstruct Pulmon Dis 15:125-134.

Roesch EA, Nichols DP, and Chmiel JF (2018) Inflammation in cystic fibrosis: an update. Pediatr Pulmonol 53:S30-S50.

Ross KR, Chmiel JF, and Konstan MW (2009) The role of inhaled corticosteroids in the management of cystic fibrosis. Paediatr Drugs 11:101-113.

Rovin BH, Dickerson JA, Tan LC, and Hebert CA (1995) Activation of nuclear factorkappa B correlates with MCP-1 expression by human mesangial cells. Kidney Int 48:1263-1271.

Ruffin M, Roussel L, Maillé É, Rousseau S, and Brochiero E (2018) Vx-809/Vx-770 treatment reduces inflammatory response to Pseudomonas aeruginosa in primary differentiated cystic fibrosis bronchial epithelial cells. Am J Physiol Lung Cell Mol Physiol 314:L635-L641.

Sagel SD, Kapsner R, Osberg I, Sontag MK, and Accurso FJ (2001) Airway inflammation in children with cystic fibrosis and healthy children assessed by sputum induction. Am J Respir Crit Care Med 164:1425-1431.

Saint-Criq V and Gray MA (2017) Role of CFTR in epithelial physiology. Cell Mol Life Sci 74:93-115.

Sasaki K and Manabe H (2004) KF19514, a phosphodiesterase 4 and 1 inhibitor, inhibits TNF-alpha-induced GM-CSF production by a human bronchial epithelial cell line via inhibition of PDE4. Inflamm Res 53:31-37.

Scarcériaux V, Pélaprat D, Forgez P, Lhiaubet AM, and Rostène W (1995) Effects of dexamethasone and forskolin on neurotensin production in rat hypothalamic cultures. Endocrinology 136:2554-2560.

Shen BQ, Finkbeiner WE, Wine JJ, Mrsny RJ, and Widdicombe JH (1994) Calu-3: a human airway epithelial cell line that shows cAMP-dependent Cl- secretion. Am J Physiol 266:L493-L501.

Shikhagaie MM, Andersson CK, Mori M, Kortekaas Krohn I, Bergqvist A, Dahl R, Ekblad E, Hoffmann HJ, Bjermer L, and Erjefält JS (2014) Mapping of TLR5 and TLR7 in central and distal human airways and identification of reduced TLR expression in severe asthma. Clin Exp Allergy 44:184-196.

Singh D, Abbott-Banner K, Bengtsson T, and Newman K (2018) The short-term bronchodilator effects of the dual phosphodiesterase 3 and 4 inhibitor RPL554 in COPD. Eur Respir J 52:1801074.

Smith S and Edwards CT (2017) Long-acting inhaled bronchodilators for cystic fibrosis. Cochrane Database Syst Rev 12:CD012102.

Tang A, Sharma A, Jen R, Hirschfeld AF, Chilvers MA, Lavoie PM, and Turvey SE (2012) Inflammasome-mediated IL-1 $\beta$ production in humans with cystic fibrosis. PLoS One 7:e37689.

Taylor DR and Hancox RJ (2000) Interactions between corticosteroids and beta agonists. Thorax 55:595-602.

Turner MJ, Luo Y, Thomas DY, and Hanrahan JW (2020) The dual phosphodiesterase 3/4 inhibitor RPL554 stimulates rare class III and IV CFTR mutants. Am J Physiol Lung Cell Mol Physiol 318:L908-L920. 
Turner MJ, Matthes E, Billet A, Ferguson AJ, Thomas DY, Randell SH, Ostrowski LE, Abbott-Banner K, and Hanrahan JW (2016) The dual phosphodiesterase 3 and 4 inhibitor RPL554 stimulates CFTR and ciliary beating in primary cultures of bronchial epithelia. Am J Physiol Lung Cell Mol Physiol 310:L59-L70.

Tyrrell J, Qian X, Freire J, and Tarran R (2015) Roflumilast combined with adenosine increases mucosal hydration in human airway epithelial cultures af ter cigarette smoke exposure. Am J Physiol Lung Cell Mol Physiol 308: L1068-L1077.

Valdivieso AG, Clauzure M, Massip-Copiz M, and Santa-Coloma TA (2016) The chloride anion acts as a second messenger in mammalian cells - modifying the expression of specific genes. Cell Physiol Biochem 38:49-64.

Velikova TV, Krasimirova E, Lazova SM, Perenovska PI, Valerieva AD, Miteva DS, Dimitrov VD, Staevska MT, Kyurkchiev DS, and Petrova G (2018) MCP-1/CCL2 in a Bulgarian cohort of children with bronchial asthma and cystic fibrosis. Arch Immunol Allergy 1:1-5.

Wainwright CE, Elborn JS, Ramsey BW, Marigowda G, Huang X, Cipolli M, Colombo C, Davies JC, De Boeck K, Flume PA, et al.; TRAFFIC Study Group; TRANSPORT
Study Group (2015) Lumacaftor-Ivacaftor in Patients with Cystic Fibrosis Homozygous for Phe508del CFTR. $N$ Engl J Med 373:220-231.

Wilson RH, Maruoka S, Whitehead GS, Foley JF, Flake GP, Sever ML, Zeldin DC Kraft M, Garantziotis S, Nakano H, et al. (2012) The Toll-like receptor 5 ligand flagellin promotes asthma by priming allergic responses to indoor allergens. Nat Med 18:1705-1710.

Zhang YL, Chen PX, Guan WJ, Guo HM, Qiu ZE, Xu JW, Luo YL, Lan CF, Xu JB, Hao Y, et al. (2018) Increased intracellular $\mathrm{Cl}^{-}$concentration promotes ongoing inflammation in airway epithelium. Mucosal Immunol 11:1149-1157.

Zhao Y and Ding C (2018) Effects of hydrocortisone on regulating inflammation, hemodynamic stability, and preventing shock in severe sepsis patients. Med Sci Monit 24:3612-3619.

Address correspondence to: Dr. Mark J. Turner, Department of Physiology, CFTRc, McGill University, 3655 Promenade Sir William Osler, Montréal, QC, Canada H3G 1Y6. E-mail: mark.turner2@mcgill.ca 